\title{
Growth of Sobolev norms for solutions of time dependent Schrödinger operators with harmonic oscillator potential
}

\author{
J.-M. Delort \\ Université Paris 13, Institut Galilée, \\ CNRS, UMR 7539, Laboratoire Analyse Géométrie et Applications \\ 99, Avenue J.-B. Clément, \\ F-93430 Villetaneuse
}

\begin{abstract}
It has been known for some time that solutions of linear Schrödinger operators on the torus, with bounded, smooth, time dependent (order zero pseudo-differential) potential, have Sobolev norms growing at most like $t^{\epsilon}$ when $t \rightarrow+\infty$ for any $\epsilon>0$. This property is proved exploiting the fact that, on the circle, successive eigenvalues of the laplacian are separated by increasing gaps (and a more involved, but similar property, for clusters of eigenvalues in higher dimension). We study here the case of solutions of $\left(i \partial_{t}-\frac{\partial^{2}}{\partial x^{2}}+x^{2}+V\right) u=0$, where $V$ is a time periodic pseudo-differential order zero perturbation. In this case, the gap between successive eigenvalues of the stationary operator is constant. We show that there are order zero potentials $V$ for which some solutions $u$ have Sobolev norms of order $s$ growing like $t^{s / 2}$ when $t \rightarrow+\infty$, i.e. $\liminf _{t \rightarrow+\infty}\left[t^{-s / 2}\|u(t, \cdot)\|_{\mathcal{H}^{s}}\right]>0$. The idea of the proof is to construct a potential which, at the classical level, pulls frequencies to higher modes, so that they will be of size $\sqrt{t}$ at time $t$. One contructs then the wanted solution passing from the classical level to the quantum one.
\end{abstract}

\section{Introduction}

Let $P_{0}$ be an elliptic self-adjoint differential operator of positive order on some Riemannian manifold $M$. Consider $t \rightarrow V(t)$ a smooth family of self-adjoint operators on $M$, or order zero, and the Schrödinger equation

$$
\left(i \partial_{t}+P_{0}+V(t)\right) u=0 .
$$

Then $\|u(t, \cdot)\|_{L^{2}}=\|u(0, \cdot)\|_{L^{2}}$. Denote by $\mathcal{H}^{s}$ the Sobolev space associated to $P_{0}$, defined when $s$ is an even integer $2 k$ by $\|u\|_{\mathcal{H}^{s}}=\|u\|_{L^{2}}+\left\|P_{0}^{k} u\right\|_{L^{2}}$. We are interested in estimating $\|u(t, \cdot)\|_{\mathcal{H}^{s}}$ when $t$ goes to infinity. If $V$ is time independent, it is immediate that $\|u(t, \cdot)\|_{\mathcal{H}^{s}}=\|u(0, \cdot)\|_{\mathcal{H}^{s}}$. Over the last fifteen years, several results have been obtained by different authors for time dependent potentials $V$, under convenient spectral assumptions on $P_{0}$ and $V$. Our aim is to

This work was partially supported by the ANR project Equa-disp.

Keywords: Growth of Sobolev norms, Schrödinger equation, Harmonic oscillator. MSC 35Q41, 35B40. 
study this question when $P_{0}$ is the harmonic oscillator in one dimension, as this is the most important case which has not been studied by preceding authors. Before presenting our results, we discuss in a relatively lengthy way known results and the methods that have been developed to prove them.

The problem of finding optimal bounds for $\|u(t, \cdot)\|_{\mathcal{H}^{s}}$ has been addressed by Nenciu [10] and Barbaroux and Joye [1], in the abstract framework of an operator $P_{0}$ and a perturbation $V(t)$ acting on elements of an Hilbert space, when the spectrum of $P_{0}$ is discrete and has increasing gaps. This means that the distinct eigenvalues $\left(\lambda_{n}\right)_{n}$ of $P_{0}$ satisfy conditions of type $\left|\lambda_{n+1}-\lambda_{n}\right| \geq c\left(\lambda_{n}+\lambda_{n+1}\right)^{\rho / 2}$ for some positive $\rho$. This assumption is satisfied for instance if $M=\mathbb{S}^{d}$ and $P_{0}=-\Delta$ (with $\lambda_{n} \sim n^{2}$ and $\rho=1 / 2$ ). If $\Pi_{n}$ denotes the spectral projector associated to the eigenvalue $\lambda_{n}$, and if one assumes that $V(t)$ is an operator obeying conditions of type $\left\|\Pi_{n} \partial_{t}^{\ell} V(t) \Pi_{n^{\prime}}\right\|_{\mathcal{L}\left(L^{2}\right)} \leq C_{N}\left\langle n-n^{\prime}\right\rangle^{-N}$ for any $N$, $\ell$, it follows from the results of [10, 1], that solutions of (1) verify $\|u(t, \cdot)\|_{\mathcal{H}^{s}} \leq C_{\epsilon}|t|^{\epsilon}$ when $t$ goes to infinity, for any $\epsilon>0$. Actually, because of the fact that $L^{2}$ norms are conserved, and since $\mathcal{H}^{s}$ spaces are stable by interpolation, such a result follows from a seemingly weaker bound $\|u(t, \cdot)\|_{\mathcal{H}^{s}} \leq C(1+|t|)^{M}$, where $M$ is some positive number independent of $s$.

Let us recall the basic idea of the proof of such a result following the method of $[10,1]$, when for instance $P_{0}$ is of order 2: One looks for an operator $Q$ such that

$$
Q^{*}\left(i \partial_{t}+P_{0}+V(t)\right) Q=i \partial_{t}+P_{0}+W(t)+\text { remainders }
$$

where the remainders are of very negative order, and where $\left[W(t), P_{0}\right]=0$ for any $t$. Clearly, any solution of $\left(i \partial_{t}+P_{0}+W(t)\right) w=0$ will satisfy $\left\|P_{0}^{k} w(t, \cdot)\right\|_{L^{2}}=\left\|P_{0}^{k} w(0, \cdot)\right\|_{L^{2}}$ for any $t \in \mathbb{R}, k \in \mathbb{N}$. Taking the remainder in the right hand side of (2) into account, one easily deduces from that bounds $\|u(t, \cdot)\|_{\mathcal{H}^{s}} \leq C(1+|t|)\|u(0, \cdot)\|_{\mathcal{H}^{s}}$ for solutions of $(1)$. The operator $Q$ is looked for as $Q=Q_{0}+Q_{1}+\cdots$, with $Q_{0}=I d, Q_{1}=Q_{1}^{\prime}+Q_{1}^{\prime \prime}$, where $Q_{1}^{\prime}$ is of order $-\rho, Q_{1}^{\prime \prime}$ of order $-2 \rho$, $\left(Q_{1}^{\prime}\right)^{*}=-Q_{1}^{\prime},\left(Q_{1}^{\prime \prime}\right)^{*}=Q_{1}^{\prime \prime}$. Decompose $V(t)=V_{\mathrm{D}}(t)+V_{\mathrm{ND}}(t)$, where $\Pi_{n} V_{\mathrm{D}} \Pi_{n^{\prime}}=0$ if $n \neq n^{\prime}$ and $\Pi_{n} V_{\mathrm{ND}} \Pi_{n}=0$ for any $n$. Then $V_{\mathrm{D}}$ will contribute to $W$ in the right hand side of (2), and $V_{\mathrm{ND}}$ will be eliminated solving equation

$$
-\left[Q_{1}^{\prime}, P_{0}\right]+V_{\mathrm{ND}}=0 .
$$

Since this equation may be written, applying the spectral projectors $\Pi_{n}$ (resp. $\Pi_{n^{\prime}}$ ) on the left (resp. right), as

$$
\left(\lambda_{n}-\lambda_{n^{\prime}}\right) \Pi_{n} Q_{1}^{\prime} \Pi_{n^{\prime}}+\Pi_{n} V_{\mathrm{ND}} \Pi_{n^{\prime}}=0\left(n \neq n^{\prime}\right),
$$

one sees that the gap condition on the $\lambda_{n}$ 's implies that $Q_{1}^{\prime}$ will be of order $-\rho$. In particular, terms in the right hand side of $(2)$ of form $-\left[Q_{1}^{\prime}, i \partial_{t}\right]$ are of order $-\rho$, so enter the construction of $Q$ at subsequent orders.

The above approach uses in an essential way the gap condition on the eigenvalues of $P_{0}$. In particular, it does not apply a priori to the case when $P_{0}=-\Delta$ on the torus $\mathbb{T}^{d}(d \geq 2)$. Nevertheless, Bourgain $[2,3]$ could prove that, if $V$ is a smooth time dependent potential bounded as well as its derivatives, solutions of $\left(i \partial_{t}-\Delta+V\right) u=0$ on $\mathbb{T}^{d}$ obey bounds $\|u(t, \cdot)\|_{\mathcal{H}^{s}} \leq$ $C_{\epsilon}(1+|t|)^{\epsilon}$ for any $\epsilon>0$. The method of proof is quite different from what we outlined 
above, and relies on reduction to a time periodic potential, and study of the eigenfunctions of the corresponding operator on $\mathbb{S}^{1} \times \mathbb{T}^{d}$. The main technical ingredient is a partition of $\mathbb{Z}^{d}$ (considered as the dual group of $\mathbb{T}^{d}$ ) in clusters, which are well separated for the distance $\left|n-n^{\prime}\right|+\left.|| n\right|^{2}-\left|n^{\prime}\right|^{2} \mid$ naturally associated to the Schrödinger operator. The same method has been applied in dimension one by Wei-Min Wang [12] to prove logarithmic bounds for the Sobolev norm of solutions of (1), when $V$ is analytic. We gave in [4] an alternative proof of the result of Bourgain, which works as well for perturbations $V$ which are pseudo-differential operators of order zero, with smooth and bounded dependence on $t$, rediscovering the method used by Nenciu and Barbaroux-Joye, that we ignored at that time (We owe to discussions with P. Duclos and S. Kuksin references to these works and to related papers). The key point is a combination of the diagonalization method outlined above with the Bourgain lemma on separated clusters, which allows one to solve again (4) with a gain $-\rho$ on the order of $Q$ versus the order of $V$. Moreover, we could extend such results to some other manifolds, like convenient surfaces of revolution. This method has been recently adapted to the case of analytic potentials by D. Fang and Q. Zhang [7] to recover the logarithmic estimates of Wang, and extend them to higher dimensions and Gevrey potentials. Remind also that Bourgain [2] constructed examples of time periodic potentials for which $\|u(t, \cdot)\|_{H^{s}}$ is bounded from below by a power of $(\log t)^{s}$. This shows that one cannot get in general uniform estimates for the Sobolev norms. On the other hand, Wei-Min Wang [14] found an explicit example of a time dependent local potential on $\mathbb{S}^{1}$ for which there is no Sobolev growth.

A natural question is to determine whether estimates of type $\|u(t, \cdot)\|_{\mathcal{H}^{s}}=O\left(t^{\epsilon}\right), t \rightarrow+\infty$ hold true when the assumption of increasing gaps $\left|\lambda_{n+1}-\lambda_{n}\right| \geq c\left(\lambda_{n}+\lambda_{n+1}\right)^{\rho / 2}$ is not satisfied with a positive $\rho$. Howland [9] obtained results on the structure of the spectrum of $i \partial_{t}+P_{0}+V(t)$ on $\mathbb{S}^{1} \times \mathbb{S}^{1}$, when $V$ is time periodic and when the eigenvalues $\lambda_{n}$ of $P_{0}$ satisfy a shrinking gap condition, like $\lambda_{n} \sim n^{\alpha}$ with $0<\alpha<1$. Duclos, Lev and Štovíček [5] have studied Sobolev bounds for solutions of such operators, under the same assumption of shrinking gap conditions. If one sets $\gamma=\frac{1-\alpha}{2}$, and if the perturbation $V(t)$ in (1) is periodic in time, small enough, and satisfies conditions of type

$$
\left\|\Pi_{n} V(t) \Pi_{n^{\prime}}\right\|_{\mathcal{L}\left(L^{2}\right)}=O\left(\left(1+|n|+\left|n^{\prime}\right|\right)^{-2 \gamma}\left\langle n-n^{\prime}\right\rangle^{-N}\right)
$$

for any $N$, they could prove $O\left(|t|^{\epsilon}\right)$-bounds for Sobolev norms. These assumptions on $V$ are verified for instance when $V$ is a pseudo-differential operator of order $-2 \gamma$ on $\mathbb{S}^{1}$. The method of proof still relies on a conjugation of type (2), except that it is done in two steps. One first decomposes the potential $V(t)$ as $\bar{V}+\widetilde{V}(t)$, where $\bar{V}$ is the time average of $V$ on a period. One eliminates $\widetilde{V}(t)$ looking for an operator $Q$ such that, instead of $(3)$,

$$
-\left[Q, i \partial_{t}+P_{0}+\bar{V}+\tilde{V}(t)\right]+\tilde{V}(t)=\text { terms of lower order. }
$$

This equation is solved taking $Q$ such that $-\left[Q, i \partial_{t}\right]+\widetilde{V}(t)=0$. Since $\widetilde{V}(t)$ is of order $-2 \gamma, Q$ will be of order $-2 \gamma$. The shrinking gap condition on $P_{0}$ implies then that $\left[Q, P_{0}\right]$ is of order $-4 \gamma$, so will contribute to the lower order terms in the right hand side. The same is true for the $[Q, \bar{V}+\widetilde{V}(t)]$ contribution.

After the first step, one is morally reduced to to an operator of the form $i \partial_{t}+P_{0}+\bar{V}$, where $\bar{V}$ is time independent. The second step of the proof of the results of [5] is to find another operator 
$Q$ such that

$$
Q^{*}\left(i \partial_{t}+P_{0}+\bar{V}\right) Q=i \partial_{t}+P_{0}+\bar{V}_{\mathrm{D}}+\text { remainders, }
$$

where $\bar{V}_{\mathrm{D}}$ commutes to $P_{0}$. The method is similar to the one used in the case of increasing gaps, i.e. finding $Q$ solving an equation of form (3). The difference is that under a shrinking gap condition, the operator $Q$ that one finds is not of order smaller than the order of $\bar{V}$. Actually, since $V$ is of order $-2 \gamma$, where $\gamma$ s related to the width of the gaps, one finds an operator $Q$ of order zero. The key point is that $Q$ is now time independent so that $\left[i \partial_{t}, Q\right]=0$, and is small since $\bar{V}$ is small. Because of that, after finding the first approximation of $Q$ to solve equation (6), one generates remainders which are of the same order as $\bar{V}$, but whose magnitude is $\epsilon^{2}$ if the magnitude of $\bar{V}$ is $\epsilon$. Iterating the process, and showing that the resulting $\epsilon$-series converges, allows one to make a reduction of (6) at infinite order i.e. to remove the remainders in the right hand side.

The results we describe so far use in an essential way that the gaps in the spectrum of $P_{0}$ are either increasing or shrinking. They do not apply to the case of the harmonic oscillator in one dimension $P_{0}=\frac{1}{2}\left(-\frac{d^{2}}{d x^{2}}+x^{2}\right)$, for which the eigenvalues are the integers (up to translation): this corresponds to the forbidden case $\alpha=1$ or $\gamma=0$ in the preceding results.

The goal of this paper is to study this problem, and to show that one may find order zero time dependent perturbations of $P_{0}$ such that there are solutions whose $\mathcal{H}^{s}$-norms grow like $t^{s / 2}$, when $t$ goes to infinity (This growth will be seen to be optimal). In other words, our results are in contrast with those of the cases of increasing or shrinking gaps, as one cannot obtain a bound for the Sobolev norms in terms of $|t|^{M}$ with a power $M$ independent of $s$.

Let us describe the approach we follow. We consider an operator

$$
i \partial_{t}+P_{0}+\mathrm{Op}^{\mathrm{W}}(V)
$$

where $P_{0}$ is the harmonic oscillator in one dimension, and $\mathrm{Op}^{\mathrm{W}}(V)$ the Weyl quantization of a time periodic order zero symbol $V$ belonging to the symbolic classes naturally associated to $P_{0}$. One first looks for an operator of order zero $Q$, such that

$$
Q^{*}\left(i \partial_{t}+P_{0}+\mathrm{Op}^{\mathrm{W}}(V)\right) Q=i \partial_{t}+P_{0}+\mathrm{Op}^{\mathrm{W}}\left(V^{Z}\right)+\text { remainders, }
$$

where $V^{Z}$ is such that $\left[i \partial_{t}+P_{0}, V^{Z}\right]=0$. The construction of $Q$ is essentially reduced to solving an equation of the from

$$
\left[i \partial_{t}+P_{0}, Q\right]+\mathrm{Op}^{\mathrm{W}}\left(V-V^{Z}\right) Q=\text { lower order operator. }
$$

Notice that the difference with the case of increasing or shrinking gaps is that in the left hand side of $(9),\left[i \partial_{t}, Q\right]$ and $\left[P_{0}, Q\right]$ are of the same order (In the results described above, one of these commutators was of lower order, so that the equation to solve was either $\left[i \partial_{t}, Q\right]=B$ or $\left[P_{0}, Q\right]=B$ for a given $\left.B\right)$. Using symbolic calculus, we easily find $V^{Z}$ with $\left[i \partial_{t}+P_{0}, V^{Z}\right]=0$ and $Q$ solving (9), so that, forgetting the remainders, the conjugation equation (8) reduces (7) to

$$
i \partial_{t}+P_{0}+\mathrm{Op}^{\mathrm{W}}\left(V^{Z}\right) .
$$


Conjugating the above operator by $e^{i t P_{0}}$, one reduces it to

$$
i \partial_{t}+\mathrm{Op}^{\mathrm{W}}(W)
$$

where $\mathrm{Op}^{\mathrm{W}}(W)=e^{-i t P_{0}} \mathrm{Op}^{\mathrm{W}}\left(V^{Z}\right) e^{i t P_{0}}$. The commutator properties of $\mathrm{Op}^{\mathrm{W}}\left(V^{Z}\right)$ and $i \partial_{t}+P_{0}$ imply that $W$ is independent of $t$. In that way, (7) is reduced to an autonomous ODE (11).

The second step of the proof is to construct a convenient $W$ and an approximate solutions $w_{a}$ of (11), whose $\mathcal{H}^{s}$-norm behaves like $t^{s / 2}$ when $t$ goes to infinity. We look for this function as $w_{a}(t, x)=e^{i \psi(t, x)} b(t, x)$ where, for large $t,\left|\partial_{x} \psi(t, x)\right| \sim \sqrt{t},|b(t, x)| \leq C t^{-1 / 4}, x \rightarrow b(t, x)$ is supported for $x / \sqrt{t}$ in a compact set $K$ and $|b(t, x)| \geq c t^{-1 / 4}$ for $x / \sqrt{t}$ in another compact set $K^{\prime}$. In that way, $\left\|w_{a}(t, \cdot)\right\|_{L^{2}} \sim 1$ and $\left\|\partial_{x}^{s} w_{a}(t, \cdot)\right\|_{L^{2}}+\left\|x^{s} w_{a}(t, \cdot)\right\|_{L^{2}} \sim t^{s / 2}$ i.e. $\left\|w_{a}(t, \cdot)\right\|_{\mathcal{H}^{s}} \sim t^{s / 2}$ when $t$ goes to infinity. To determine $\psi$, we construct the Lagrangian

$$
\Lambda_{t}=\left\{\left(x, \partial_{x} \psi(t, x)\right) ; x \in \mathbb{R}\right\}
$$

At the initial time, we take a Lagrangian which is a graph above an interval $[A, 2 A]$, and which stays inside an angular sector of angle $1 / A$ around the positive $x$-axis of $\mathbb{R}^{2} \simeq T^{*} \mathbb{R}$, where $A$ is a large enough constant. We take an autonomous symbol $W$ that may be written in polar coordinates as $W_{\infty}(\theta)+$ perturbation of order $<-2$. The main contribution $W_{\infty}$ is assumed to satisfy convenient non-degeneracy conditions along the positive $x$-axis $\theta=0$. These conditions imply that the flow-out of the Hamiltonian vector field of $W$ pulls the initial Lagrangian far away from zero, inside the sector of angle $1 / A$. At large time $t$, the transported Lagrangian lies at a distance of order $\sqrt{t}$ from the $x$-axis, and is the graph of a function $x \rightarrow \partial_{x} \psi(t, x)$, which has thus to verify $\left|\partial_{x} \psi(t, x)\right| \sim \sqrt{t}$. Once $\psi$ has been constructed, one finds $b$ so that $e^{i \psi} b$ be an approximate solution, solving transport equations. In that way, an approximate solution of $\left(i \partial_{t}+\mathrm{Op}^{\mathrm{W}}(W)\right) w_{a}=0$ has been constructed, for some convenient $W$. The last step of the proof is to show that one may find a time depending symbol $V$ such that the reductions (8), (10), (11) bring to this $W$ (up to remainders), and to deduce from the approximate solution $w_{a}$ an approximate solution - and then an exact solution - of the initial operator (7), enjoying the wanted asymptotics at infinity.

\section{Growth of Sobolev norms}

\subsection{Statement of the main theorem}

The main result of this paper asserts that one may construct time dependent self-adjoint order zero perturbations of the harmonic oscillator $P_{0}=\frac{1}{2}\left(-\frac{d^{2}}{d x^{2}}+x^{2}\right)$ in one space dimension, with solutions whose Sobolev norm grows at an optimal rate when time goes to infinity. Before stating the theorem, we need to introduce some notations.

Let us recall the definition of standard classes of pseudo-differential symbols adapted to the harmonic oscillator. 
Definition 1.1.1 Let $d \in \mathbb{N}^{*}, m \in \mathbb{R}$. One denotes by $\mathcal{S}^{m}\left(\mathbb{S}^{1} \times \mathbb{R}^{d} \times \mathbb{R}^{d}\right)$ the space of smooth functions $(t, x, \xi) \rightarrow a(t, x, \xi)$ defined on $\mathbb{R} \times \mathbb{R}^{d} \times \mathbb{R}^{d}$, with values in $\mathbb{C}$, which are $2 \pi$-periodic in $t$, and such that for any $j, \alpha, \beta \in \mathbb{N}$, there is a constant $C>0$ so that, for any $(t, x, \xi)$,

$$
\left|\partial_{t}^{j} \partial_{x}^{\alpha} \partial_{\xi}^{\beta} a(t, x, \xi)\right| \leq C(1+|x|+|\xi|)^{m-|\alpha|-|\beta|} .
$$

We endow $\mathcal{S}^{m}\left(\mathbb{S}^{1} \times \mathbb{R}^{d} \times \mathbb{R}^{d}\right)$ with its natural semi-norms. We denote by $\mathcal{S}^{m}\left(\mathbb{R}^{d} \times \mathbb{R}^{d}\right)$ the subspace of time independent symbols.

We shall use Weyl quantization for the above symbols.

Definition 1.1.2 If $a \in \mathcal{S}^{m}\left(\mathbb{S}^{1} \times \mathbb{R}^{d} \times \mathbb{R}^{d}\right)$, we denote by $\mathrm{Op}^{\mathrm{W}}(a(t, \cdot))$ the operator defined on $\mathcal{S}\left(\mathbb{R}^{d}\right)$ by

$$
\mathrm{Op}^{\mathrm{W}}(a(t, \cdot)) u=\frac{1}{(2 \pi)^{d}} \int e^{i(x-y) \xi} a\left(t, \frac{x+y}{2}, \xi\right) u(y) d y d \xi
$$

We refer to chapter 4 of the book of Shubin [11] for the main properties of these operators. We shall recall those which will be useful for us in subsection 1.2 below. Let us just mention at this point that if $a \in \mathcal{S}^{0}\left(\mathbb{S}^{1} \times \mathbb{R}^{d} \times \mathbb{R}^{d}\right)$, for fixed $t, \mathrm{Op}^{\mathrm{W}}(a(t, \cdot))$ extends as a bounded operator on $L^{2}\left(\mathbb{R}^{d}\right)$ and on $\mathcal{S}^{\prime}\left(\mathbb{R}^{d}\right)$. Moreover, if one defines, for $s \in \mathbb{R}$, the Sobolev space $\mathcal{H}^{s}$ associated to the harmonic oscillator, by

$$
\mathcal{H}^{s}\left(\mathbb{R}^{d}\right)=\left\{u \in \mathcal{S}^{\prime}\left(\mathbb{R}^{d}\right) ; \mathrm{Op}^{\mathrm{W}}\left(\left(1+|x|^{2}+|\xi|^{2}\right)^{s / 2}\right) u \in L^{2}\left(\mathbb{R}^{d}\right)\right\}
$$

or equivalently, when $s \in \mathbb{N}$,

(1.1.4) $\mathcal{H}^{s}\left(\mathbb{R}^{d}\right)=\left\{u \in \mathcal{S}^{\prime}\left(\mathbb{R}^{d}\right)\right.$; for any $\alpha, \beta \in \mathbb{N}^{d} \times \mathbb{N}^{d}$ with $\left.|\alpha|+|\beta| \leq s, x^{\alpha} \partial^{\beta} u \in L^{2}\left(\mathbb{R}^{d}\right)\right\}$,

the operator $\mathrm{Op}^{\mathrm{W}}(a(t, \cdot))$ is bounded from $\mathcal{H}^{s}\left(\mathbb{R}^{d}\right)$ to $\mathcal{H}^{s-m}\left(\mathbb{R}^{d}\right)$, uniformly in $t$, for any $s$, when $a$ is a symbol of order $m$.

Let state our main theorem.

Theorem 1.1.3 Let $d=1$. There is a real valued symbol $V \in \mathcal{S}^{0}\left(\mathbb{S}^{1} \times \mathbb{R} \times \mathbb{R}\right)$ and, for any $s>0$, a constant $c>0$, and a smooth solution $u: \mathbb{R} \times \mathbb{R} \rightarrow \mathbb{C}$ of the equation

$$
\left(i \partial_{t}+P_{0}+\mathrm{Op}^{\mathrm{W}}(V(t, \cdot))\right) u=0,
$$

such that for any $t \geq 0, u(t, \cdot) \in \mathcal{H}^{s}(\mathbb{R})$ and satisfies

$$
\|u(t, \cdot)\|_{\mathcal{H}^{s}} \geq c t^{s / 2}
$$

for any large enough $t$. 
Remarks: - We shall see, in the following subsection, that a solution of equation (1.1.5) with any $V$ of order zero and with $\mathcal{H}^{s}$-Cauchy data, always satisfies a bound $\|u(t, \cdot)\|_{\mathcal{H}^{s}} \leq C t^{s / 2}$ when $t \rightarrow+\infty$. The lower bound (1.1.6) is thus optimal.

- The symbol $V$ that we construct will be an element of $\mathcal{S}^{0}\left(\mathbb{S}^{1} \times \mathbb{R} \times \mathbb{R}\right)$. In particular, it depends on $x$ and on $\xi$. We do not know if a result of type (1.1.6) could be proved replacing in (1.1.5) the order zero perturbation $\mathrm{Op}^{\mathrm{W}}(V(t, \cdot))$ by a local potential $V(t, x)$. On the other hand, Grébert and Thomann [8] have studied recently equations of the form (1.1.5), where $V$ is a small time quasi-periodic local potential satisfying convenient assumptions. They have been able to prove that, when the parameter of quasi-periodicity stays outside a subset of small measure, the equation may be reduced to a linear equation with constant coefficients. Consequently, the solutions of the Cauchy problem are time quasi-periodic, and so have uniformly bounded Sobolev norms. We refer to theorem 1.2 and corollary 1.3 of [8] for precise statements, and to corollary 1.4 of the same paper, as well as to the paper of Wang [13], for interpretation of such results in terms of Floquet spectrum. Notice also that similar results for the Schrödinger equation on the torus, with time quasi-periodic potential, had been previously proved by Eliasson and Kuksin [6].

- The conditions on the potential that we need to obtain (1.1.6) will be made more precise in subsection 3.1 .

\subsection{Symbolic calculus}

In this subsection, we recall classical properties of operators of the form (1.1.2) and establish some related results that will be useful in the sequel.

Remind that if $a$ is in $\mathcal{S}^{m}\left(\mathbb{S}^{1} \times \mathbb{R}^{d} \times \mathbb{R}^{d}\right)$, then

$$
\mathrm{Op}^{\mathrm{W}}(a(t, \cdot))^{*}=\mathrm{Op}^{\mathrm{W}}(\bar{a}(t, \cdot)),
$$

so that real valued symbols give rise to self-adjoint operators. If $a \in \mathcal{S}^{m_{1}}\left(\mathbb{S}^{1} \times \mathbb{R}^{d} \times \mathbb{R}^{d}\right)$, $b \in \mathcal{S}^{m_{2}}\left(\mathbb{S}^{1} \times \mathbb{R}^{d} \times \mathbb{R}^{d}\right)$, there is a symbol $c \in \mathcal{S}^{m_{1}+m_{2}}\left(\mathbb{S}^{1} \times \mathbb{R}^{d} \times \mathbb{R}^{d}\right)$ such that

$$
\mathrm{Op}^{\mathrm{W}}(a(t, \cdot)) \circ \mathrm{Op}^{\mathrm{W}}(b(t, \cdot))=\mathrm{Op}^{\mathrm{W}}(c(t, \cdot)) .
$$

Moreover, there is a sequence $\left(c_{k}\right)_{k}$ of symbols, $c_{k} \in \mathcal{S}^{m_{1}+m_{2}-2 k}\left(\mathbb{S}^{1} \times \mathbb{R}^{d} \times \mathbb{R}^{d}\right)$, such that $c-\sum_{k=0}^{N-1} c_{k} \in \mathcal{S}^{m_{1}+m_{2}-2 N}\left(\mathbb{S}^{1} \times \mathbb{R}^{d} \times \mathbb{R}^{d}\right)$, where

$$
c_{k}(t, x, \xi)=2^{-k} \sum_{|\alpha|+|\beta|=k} \frac{(-1)^{|\beta|}}{\alpha ! \beta !}\left(\partial_{\xi}^{\alpha} D_{x}^{\beta} a\right)\left(\partial_{\xi}^{\beta} D_{x}^{\alpha} b\right) .
$$

The above expression is bilinear in $(a, b)$ and symmetric (resp. antisymmetric) if $k$ is even (resp. odd). This implies that

$$
\left[\mathrm{Op}^{\mathrm{W}}(a(t, \cdot)), \mathrm{Op}^{\mathrm{W}}(b(t, \cdot))\right]=\mathrm{Op}^{\mathrm{W}}(e(t, \cdot)),
$$


where $e \in \mathcal{S}^{m_{1}+m_{2}-2}\left(\mathbb{S}^{1} \times \mathbb{R}^{d} \times \mathbb{R}^{d}\right)$ is such that there is a sequence $\left(e_{2 k+1}\right)_{k}$ of symbols, with $e_{2 k+1} \in \mathcal{S}^{m_{1}+m_{2}-4 k-2}\left(\mathbb{S}^{1} \times \mathbb{R}^{d} \times \mathbb{R}^{d}\right)$, and

$$
\begin{aligned}
& e(t, x, \xi)-\sum_{k=0}^{N-1} e_{2 k+1}(t, x, \xi) \in \mathcal{S}^{m_{1}+m_{2}-4 N-2}\left(\mathbb{S}^{1} \times \mathbb{R}^{d} \times \mathbb{R}^{d}\right) \\
& e_{1}(t, x, \xi)=i\{a, b\}=i \sum_{j=1}^{d}\left(-\frac{\partial a}{\partial \xi_{j}} \frac{\partial b}{\partial x_{j}}+\frac{\partial a}{\partial x_{j}} \frac{\partial b}{\partial \xi_{j}}\right) .
\end{aligned}
$$

We set $p_{0}(x, \xi)=\frac{1}{2}\left(|x|^{2}+|\xi|^{2}\right)$. If $a \in \mathcal{S}^{m}\left(\mathbb{S}^{1} \times \mathbb{R}^{d} \times \mathbb{R}^{d}\right)$ we get

$$
\begin{gathered}
P_{0}=\mathrm{Op}^{\mathrm{W}}\left(p_{0}\right)=-\frac{1}{2} \Delta+\frac{1}{2}|x|^{2}, \\
{\left[P_{0}, \mathrm{Op}^{\mathrm{W}}(a(t, \cdot))\right]=i \mathrm{Op}^{\mathrm{W}}\left(\left\{p_{0}, a\right\}(t, \cdot)\right) .}
\end{gathered}
$$

The above properties of symbolic calculus provide immediately a bound for the Sobolev norm of solutions of equations of form (1.1.5):

Lemma 1.2.1 Let $V \in \mathcal{S}^{0}\left(\mathbb{S}^{1} \times \mathbb{R}^{d} \times \mathbb{R}^{d}\right)$ a real valued symbol. If $t, t^{\prime} \in \mathbb{R}$, denote by $S\left(t, t^{\prime}\right)$ the operator solving

$$
\begin{aligned}
{\left[i \partial_{t}+P_{0}+\mathrm{Op}^{\mathrm{W}}(V(t, \cdot))\right] S\left(t, t^{\prime}\right) } & =0 \\
\left.S\left(t, t^{\prime}\right)\right|_{t=t^{\prime}} & =I d .
\end{aligned}
$$

Then, for any $s \in \mathbb{R}_{+}$, there is a constant $C_{s}>0$ such that, for any $t, t^{\prime} \in \mathbb{R}$,

$$
\left\|S\left(t, t^{\prime}\right)\right\|_{\mathcal{L}\left(\mathcal{H}^{s}, \mathcal{H}^{s}\right)} \leq C_{s}\left\langle t-t^{\prime}\right\rangle^{s / 2} .
$$

In particular, any solution $u$ of (1.1.5) with initial data in $\mathcal{H}^{s}$ satisfies $\|u\|_{\mathcal{H}^{s}}=O\left(t^{s / 2}\right), t \rightarrow$ $+\infty$

Proof: Consider $w$ solution of

$$
\begin{aligned}
{\left[i \partial_{t}+P_{0}+\mathrm{Op}^{\mathrm{W}}(V(t, \cdot))\right] w(t, \cdot) } & =0 \\
w\left(t^{\prime}, x\right) & =w_{0} .
\end{aligned}
$$

Then, since $\mathrm{Op}^{\mathrm{W}}(V(t, \cdot))$ is self-adjoint on $L^{2},\|w(t, \cdot)\|_{L^{2}}=\left\|w_{0}\right\|_{L^{2}}$. Moreover, if $k \in \mathbb{N}$, it follows from (1.2.6) that

$$
\left[i \partial_{t}+P_{0}+\mathrm{Op}^{\mathrm{W}}(V(t, \cdot))\right]\left(P_{0}^{k} w\right)=g
$$

where $\|g\|_{L^{2}} \leq C\|w(t, \cdot)\|_{\mathcal{H}^{2 k-2}}$. It follows by induction that

$$
\|w(t, \cdot)\|_{\mathcal{H}^{2 k}} \leq C_{k}\left\langle t-t^{\prime}\right\rangle^{k}\left\|w\left(t^{\prime}, \cdot\right)\right\|_{\mathcal{H}^{2 k}} .
$$

Inequality (1.2.8) follows for any positive $s$ by interpolation.

In section 3 , we shall study the action of operators of the form (1.1.2) (with $d=1$ ) on some functions, given by the product of an oscillating term and a compactly supported profile. The classes of profiles we shall use is defined below. 
Definition 1.2.2 Let $j \in \mathbb{N}, t \rightarrow \mu_{\ell}(t), \ell=1,2$ be two continuous functions from $[2,+\infty[$ to $\mathbb{R}_{+}^{*}$ such that $\mu_{1}(t)<\mu_{2}(t)$ for any $t \geq 2$. Set $K(t)=\left[\mu_{1}(t), \mu_{2}(t)\right]$ and $K=(K(t))_{t \geq 2}$. One denotes by $\Sigma_{K}^{-j}$ the space of smooth functions $(t, x) \rightarrow b(t, x)$, defined on $[2,+\infty[\times \mathbb{R}$, with values in $\mathbb{C}$, such that for any $t \geq 2, x \rightarrow b(t, x)$ is supported for $\frac{x}{\sqrt{t}} \in K(t)$ and satisfies, for any $\alpha, \beta \in \mathbb{N}$, estimates

$$
\left|\partial_{t}^{\alpha} \partial_{x}^{\beta} b(t, x)\right| \leq C_{\alpha, \beta} t^{-\frac{1}{4}-\alpha-\frac{\beta}{2}-j}(\log t)^{\alpha+\beta+2 j}
$$

for any $t \geq 2, x \in \mathbb{R}$.

We assume from now on that we are given two positive real numbers $\mu_{1}^{\prime}<\mu_{2}^{\prime}$ and $\nu>0$ such that, for any $t \geq 2, \mu_{1}^{\prime}+\nu \leq \mu_{1}(t)<\mu_{2}(t) \leq \mu_{2}^{\prime}-\nu$. Let $(t, x) \rightarrow \psi(t, x)$ be a smooth real valued function, such that $Z(t, y)=\frac{1}{\sqrt{t}}\left(\partial_{x} \psi\right)(t, \sqrt{t} y)$ is defined on $\left[2,+\infty\left[\times\left[\mu_{1}^{\prime}, \mu_{2}^{\prime}\right]\right.\right.$ and satisfies for any $(t, y)$

$$
\left|\partial_{t}^{\alpha} \partial_{y}^{\beta} Z(t, y)\right| \leq C_{\alpha, \beta} t^{-\alpha}
$$

Proposition 1.2.3 Assume that there is $c>0$ such that $|Z(t, y)| \geq c$ for any $t \geq 2$, any $y \in\left[\mu_{1}^{\prime}, \mu_{2}^{\prime}\right]$. Let $m \in \mathbb{R}, N \in \mathbb{N}, j \in \mathbb{N}, a \in \mathcal{S}^{m}(\mathbb{R} \times \mathbb{R})$. There is, for any $j^{\prime} \in \mathbb{N}$, a linear map $L: \Sigma_{K}^{-j^{\prime}} \rightarrow \Sigma_{K}^{-j^{\prime}}$ and, for any $b \in \Sigma_{K}^{-j}$, there is a smooth function $r:[2,+\infty[\times \mathbb{R} \rightarrow \mathbb{C}$, satisfying for any $\alpha, \beta \in \mathbb{N}$ estimates

$$
\left|\partial_{t}^{\alpha} \partial_{x}^{\beta} r(t, x)\right| \leq C_{\alpha \beta} t^{-\frac{1}{4}-\alpha-\frac{\beta}{2}-N-1}\langle x / \sqrt{t}\rangle^{-N}(\log t)^{\alpha+\beta},
$$

such that

$$
\begin{aligned}
\mathrm{Op}^{\mathrm{W}}(a)\left[e^{i \psi(t, x)} b(t, x)\right]= & e^{i \psi(t, x)}\left[a\left(x, \partial_{x} \psi(t, x)\right) b(t, x)\right. \\
& -i\left(\partial_{\xi} a\right)\left(x, \partial_{x} \psi(t, x)\right) \partial_{x} b(t, x) \\
& \left.-\frac{i}{2}\left(\left(\partial_{x} \partial_{\xi} a\right)\left(x, \partial_{x} \psi(t, x)\right)+\left(\partial_{\xi}^{2} a\right)\left(x, \partial_{x} \psi(t, x)\right) \partial_{x}^{2} \psi(t, x)\right) b(t, x)\right] \\
& -i e^{i \psi(t, x)} \frac{(\log t)^{2}}{t^{2-\frac{m}{2}}} L(b)(t, x)+e^{i \psi(t, x)} r(t, x) .
\end{aligned}
$$

Proof: Write the left hand side of (1.2.12)

$$
T(x)=\frac{1}{2 \pi} \int e^{i(x-y) \xi} a\left(\frac{x+y}{2}, \xi\right) e^{i \psi(t, y)} b(t, y) d y d \xi .
$$

Define $B(t, y)=b(t, \sqrt{t} y)$ and compute

$$
T(x \sqrt{t})=\frac{t}{2 \pi} \int e^{i t(x-y) \xi+i \psi(t, y \sqrt{t})} a\left(\sqrt{t}\left(\frac{x+y}{2}\right), \sqrt{t} \xi\right) B(t, y) d y d \xi .
$$

Notice first that for $|x|$ large enough, $\partial_{x}^{\alpha}[T(x \sqrt{t})]=O\left(t^{-\frac{M}{2}}|x|^{-M}\right)$ for any $\alpha, M$. Actually, since

$$
\begin{aligned}
\partial_{x}^{\alpha} \partial_{\xi}^{\beta}\left[a\left(\sqrt{t}\left(\frac{x+y}{2}\right), \sqrt{t} \xi\right)\right] & =O\left(t^{\frac{m_{+}+\alpha+\beta}{2}}(1+|x|+|\xi|)^{m_{+}}\right), \\
\left|t \xi-\sqrt{t}\left(\partial_{x} \psi\right)(t, y \sqrt{t})\right| & =t|\xi-Z(t, y)|,
\end{aligned}
$$


and since $y$ stays in $\left[\mu_{1}^{\prime}, \mu_{2}^{\prime}\right]$, performing, for large $|\xi|, \partial_{y}$-integrations by parts, makes appear a $O\left((\sqrt{t}|\xi|)^{-M}\right)$ decay inside the integral in (1.2.14). Moreover, $\partial_{\xi}$-integrations by parts provide a gain $O\left((\sqrt{t}|x|)^{-M}\right)$ for large $x$. This shows that for $x$ large enough, (1.2.14) and its derivatives are bounded by $C_{M} t^{-M / 2}|x|^{-M}$ for any $M$. Returning to the original variables, we get a contribution to $r$ in (1.2.12). We may therefore assume from now on that $x$ in (1.2.14) satisfies $|x| \leq C$ for a fixed constant $C$. We perform the following change of variables

$$
y=x-z, \xi=\frac{1}{\sqrt{t}}\left(\frac{\partial \psi}{\partial x}\right)\left(t, \sqrt{t}\left(x-\frac{z}{2}\right)\right)+\eta=Z\left(t, x-\frac{z}{2}\right)+\eta .
$$

The phase in (1.2.14) may be expanded, using the expression of $\partial_{x} \psi$ in terms of $Z$, as

$$
t z\left(\eta+Z\left(t, x-\frac{z}{2}\right)\right)+\psi(t, \sqrt{t}(x-z))=\psi(t, \sqrt{t} x)+t z \eta+t z^{3} g(t, x, z),
$$

where $g$ is smooth and satisfies estimates of type $\partial_{t}^{\alpha} \partial_{x}^{\beta} \partial_{z}^{\gamma} g=O\left(t^{-\alpha}\right)$. We rewrite (1.2.14) as $(1.2 .15)$

$$
T(x \sqrt{t})=\frac{t}{2 \pi} e^{i \psi(t, x \sqrt{t})} \int e^{i t\left[z \eta+z^{3} g(t, x, z)\right]} a\left(\sqrt{t}\left(x-\frac{z}{2}\right), \sqrt{t}\left(Z\left(t, x-\frac{z}{2}\right)+\eta\right)\right) B(t, x-z) d z d \eta .
$$

Let us write

$$
a\left(\sqrt{t}\left(x-\frac{z}{2}\right), \sqrt{t}\left(Z\left(t, x-\frac{z}{2}\right)+\eta\right)\right)=\sum_{k=0}^{M}(\sqrt{t} \eta)^{k} a_{k}\left(t, x-\frac{z}{2}, \eta\right),
$$

where $a_{0}, \ldots, a_{M-1}$ are independent of $\eta$ and

$$
\begin{aligned}
a_{k}\left(t, x-\frac{z}{2}\right) & =\frac{1}{k !}\left(\partial_{\xi}^{k} a\right)\left(\sqrt{t}\left(x-\frac{z}{2}\right), \sqrt{t} Z\left(t, x-\frac{z}{2}\right)\right), 0 \leq k \leq M-1, \\
a_{M}\left(t, x-\frac{z}{2}, \eta\right) & =\frac{1}{(M-1) !} \int_{0}^{1}\left(\partial_{\xi}^{M} a\right)\left(\sqrt{t}\left(x-\frac{z}{2}\right), \sqrt{t} Z\left(t, x-\frac{z}{2}\right)+\sqrt{t} \sigma \eta\right)(1-\sigma)^{M-1} d \sigma .
\end{aligned}
$$

Decompose from (1.2.16), $T(x \sqrt{t})=\sum_{k=0}^{M} T_{k}(x \sqrt{t})$. For $0 \leq k \leq M-1$

$$
T_{k}(x \sqrt{t})=\left.e^{i \psi(t, x \sqrt{t})}\left(\frac{i}{\sqrt{t}}\right)^{k} \partial_{z}^{k}\left[e^{i t z^{3} g} a_{k}\left(t, x-\frac{z}{2}\right) B(t, x-z)\right]\right|_{z=0}
$$

The contributions coming from $k=0$ or 1 give, returning to the original variables, the main contributions to the right hand side of (1.2.12). Let us show that for $k=3, \ldots, M-1$, we get expressions that may be incorporated to the $L(b)$ term in (1.2.12). Notice that every time a $\partial_{z}$-derivative acts on the exponential in (1.2.18), we have to make act at least two more derivatives on $\partial_{z}\left[i t z^{3} g\right]$ if we want to get a non-vanishing term. Consequently, (1.2.18) is a linear combination of quantities

$$
e^{i \psi(t, x \sqrt{t})}\left(\frac{i}{\sqrt{t}}\right)^{k} t^{\ell} h_{k}(t, x),
$$

where $3 \ell \leq k$ and $h_{k}$ is computed from derivatives of order smaller than $k-3 \ell$ of $a_{k}(t, x-$ $z / 2) B(t, x-z)$ taken at $z=0$. The logarithmic losses coming, because of the derivatives, from 
$B(t, x)=b(t, \sqrt{t} x)$ will be at most $(\log t)^{k-3 \ell}$. Moreover, the expression of $a_{k}$, estimates (1.1.1), and the fact that $\sqrt{t} x$ and $\sqrt{t} Z(t, x)$ are of size $\sqrt{t}$ when $x$ is in the support of $B(t, \cdot)$, show that

$$
t^{\ell-\frac{k}{2}}\left|h_{k}(t, x)\right| \leq C t^{\ell-k}(\log t)^{k-3 \ell} t^{-\frac{1}{4}-j+\frac{m}{2}}(\log t)^{2 j} .
$$

The conditions $3 \ell \leq k, k \geq 2, k, \ell \in \mathbb{N}$, show that the prefactor $t^{\ell-k}(\log t)^{k-3 \ell}$ is always smaller than $C t^{-2}(\log t)^{2}$. Since, moreover, $\partial_{x}\left(\right.$ resp. $\left.\partial_{t}\right)$ derivatives of $h_{k}$ give a loss of $\log t$ (resp. a gain of $t^{-1}$ and a $\operatorname{loss}$ of $\log t$ ), we conclude, returning to the original variables, that (1.2.18) gives to the right hand side of (1.2.12) a contribution of form $e^{i \psi(t, x)} \frac{(\log t)^{2}}{t^{2-\frac{m}{2}}} c$, where $c$ is a local linear expression in terms of $b$ and its derivatives, belonging to $\Sigma_{K}^{-j}$ when $b$ is in $\Sigma_{K}^{-j}$.

We are left with studying $T_{M}$. Let $\chi \in C_{0}^{\infty}\left(\mathbb{R}^{2}\right)$ with small support, equal to one close to zero. Write

$$
T_{M}(x \sqrt{t})=\frac{t}{2 \pi} e^{i \psi(t, \sqrt{t} x)} \int e^{i t\left[z \eta+z^{3} g(t, x, z)\right]}(\sqrt{t} \eta)^{M} \chi(z, \eta) a_{M}\left(t, x-\frac{z}{2}, \eta\right) B(t, x-z) d z d \eta+\tilde{r}(t, x),
$$

where $\tilde{r}$ is defined by a similar integral, with $\chi$ replaced by $1-\chi$. In particular, the support of the integrand in the expression of $\tilde{r}$ does not contain $(0,0)$, which is the only critical point of the phase $z \eta+z^{3} g(t, x, z)$. Taking into account that a $\partial_{z}$ or $\partial_{\eta}$ integration by parts makes gain $t^{-1}$ and lose at most $\sqrt{t}$, we conclude that $\tilde{r}=O\left(t^{-\infty}\right)$ as well as its derivatives (Notice that for large $|\eta|, \partial_{z}$-integrations by parts provide a gain of $(\sqrt{t}|\eta|)^{-M^{\prime}}$ for any $M^{\prime}$, which makes converge the $\eta$-integral). Consequently, $\tilde{r}$ contributes to $r$ in (1.2.12), after returning to the original coordinates, since we have already reduced ourselves to the case of bounded $x$ in (1.2.14).

To conclude the proof, let us show that the first term in the right hand side of (1.2.20) contributes to $r$ in (1.2.12), up to the change of variables $x \rightarrow x / \sqrt{t}$. Rewrite this quantity as the product of $e^{i \psi(t, \sqrt{t} x)}$ and

$$
I=\frac{1}{2 \pi} \int e^{i t z \eta} t^{1+\frac{M}{2}}\left(\eta-z^{2} g(t, x, z)\right)^{M} c(t, x, z, \eta) d z d \eta
$$

with

$$
c(t, x, z, \eta)=\chi\left(z, \eta-z^{2} g(t, x, z)\right) a_{M}\left(t, x-\frac{z}{2}, \eta-z^{2} g\right) B(t, x-z) .
$$

If Supp $\chi$ is small enough, it follows from (1.1.1), (1.2.9) and (1.2.17), and from the fact that $x-\frac{z}{2}$ and $Z\left(t, x-\frac{z}{2}\right)$ stay between two positive constants in the expression of $a_{M}$, that

$$
\left|\partial_{t}^{\alpha} \partial_{x}^{\beta} \partial_{z}^{\gamma} \partial_{\eta}^{\delta}[c(t, x, z, \eta)]\right| \leq C t^{-\frac{M}{2}-\alpha+\frac{m}{2}-\frac{1}{4}-j}(\log t)^{\alpha+\beta+\gamma+2 j} .
$$

We may perform in (1.2.21) $M$ integrations by parts in $\partial_{z}$ or $\partial_{\eta}$ to get

$$
\left|\partial_{t}^{\alpha} \partial_{x}^{\beta} I\right| \leq C t^{1-M-\alpha+\frac{m}{2}-\frac{1}{4}-j}(\log t)^{\alpha+\beta+2 j+M} .
$$

If we remember that we reduced ourselves to $x$ remaining in a compact subset, we see, returning to the original variables, that we get estimates of form (1.2.11), if $M$ has been chosen large enough relatively to $N$. This concludes the proof. 


\section{Reduction to an autonomous equation}

The goal of this section is to reduce by conjugation equation (1.1.5) to an autonomous one. We study, in the first subsection, an homological equation on symbols, that will be the key of the arguments of the second subsection. Since our reduction works in any dimension, we present the arguments for a $d$-dimensional harmonic oscillator.

\section{$2.1 \quad$ Homological equation on symbols}

Let us define subspaces of the class of symbols $\mathcal{S}^{m}\left(\mathbb{S}^{1} \times \mathbb{R}^{d} \times \mathbb{R}^{d}\right)$ of definition 1.1.1.

Definition 2.1.1 Set for $m \in \mathbb{R}$

$$
\begin{aligned}
\mathcal{S}_{Z}^{m}\left(\mathbb{S}^{1} \times \mathbb{R}^{d} \times \mathbb{R}^{d}\right)=\left\{a \in \mathcal{S}^{m}\left(\mathbb{S}^{1} \times \mathbb{R}^{d} \times \mathbb{R}^{d}\right) ; \partial_{t} a+\left\{p_{0}, a\right\}=0\right\} \\
\mathcal{S}_{B}^{m}\left(\mathbb{S}^{1} \times \mathbb{R}^{d} \times \mathbb{R}^{d}\right)=\left\{a \in \mathcal{S}^{m}\left(\mathbb{S}^{1} \times \mathbb{R}^{d} \times \mathbb{R}^{d}\right) ; \text { there is } b \in \mathcal{S}^{m}\left(\mathbb{S}^{1} \times \mathbb{R}^{d} \times \mathbb{R}^{d}\right)\right. \\
\text { with } \left.a=\partial_{t} b+\left\{p_{0}, b\right\}\right\}
\end{aligned}
$$

For $t \in \mathbb{R}$, denote by $\rho(t)$ the rotation of angle $t$ acting on each couple of variables $\left(x_{j}, \xi_{j}\right)$ of $T^{*} \mathbb{R}^{d}=\mathbb{R}^{d} \times \mathbb{R}^{d}$. In complex coordinates

$$
\rho(t) \cdot\left(x_{1}+i \xi_{1}, \ldots, x_{d}+i \xi_{d}\right)=e^{i t}\left(x_{1}+i \xi_{1}, \ldots, x_{d}+i \xi_{d}\right) .
$$

Notice that $\dot{\rho}(t)=\rho(t) J$, where $J$ is the $(2 k) \times(2 k)$ block diagonal matrix whose diagonal blocks are $\left[\begin{array}{cc}0 & -1 \\ 1 & 0\end{array}\right]$.

If $a \in \mathcal{S}^{m}\left(\mathbb{S}^{1} \times \mathbb{R}^{d} \times \mathbb{R}^{d}\right)$, define

$$
M_{0}(a)(t, x, \xi)=\frac{1}{2 \pi} \int_{0}^{2 \pi} a(s+t, \rho(s) \cdot(x, \xi)) d s .
$$

Notice that if $a_{0}=M_{0}(a)$,

$\partial_{t} a_{0}+\left\{p_{0}, a_{0}\right\}=\frac{1}{2 \pi} \int_{0}^{2 \pi}\left(\partial_{t} a\right)(s+t, \rho(s) \cdot(x, \xi)) d s+\frac{1}{2 \pi} \int_{0}^{2 \pi}(D a)(s+t, \rho(s) \cdot(x, \xi)) \cdot \rho(s) J \cdot(x, \xi) d s$,

where $D a$ is differentiation with respect to the variables of $\mathbb{R}^{d} \times \mathbb{R}^{d}$. Performing an integration by parts in the first integral, and using that $\dot{\rho}=\rho J$, we obtain $\partial_{t} a_{0}+\left\{p_{0}, a_{0}\right\}=0$ i.e. $M_{0}(a) \in$ $\mathcal{S}_{Z}^{m}\left(\mathbb{S}^{1} \times \mathbb{R}^{d} \times \mathbb{R}^{d}\right)$.

Proposition 2.1.2 For any $a \in \mathcal{S}^{m}\left(\mathbb{S}^{1} \times \mathbb{R}^{d} \times \mathbb{R}^{d}\right)$, there is $b \in \mathcal{S}^{m}\left(\mathbb{S}^{1} \times \mathbb{R}^{d} \times \mathbb{R}^{d}\right)$ such that

$$
\partial_{t} b+\left\{p_{0}, b\right\}=a-M_{0}(a)
$$

(i.e. $a-M_{0}(a) \in \mathcal{S}_{B}^{m}\left(\mathbb{S}^{1} \times \mathbb{R}^{d} \times \mathbb{R}^{d}\right)$ ). Moreover, the semi-norms of b are estimated in terms of those of $a$, and $b$ is real valued if $a$ is real valued. 
Proof: Set $\tilde{a}=a-M_{0}(a)$ and define

$$
b(t, x, \xi)=\int_{0}^{t} \tilde{a}(s, \rho(s-t) \cdot(x, \xi)) d s .
$$

Then $b$ solves (2.1.4) and satisfies estimates (1.1.1). We just have to check that $b$ is $2 \pi$-periodic in time. Since $\rho$ is $2 \pi$-periodic, we need to check that

$$
\int_{0}^{2 \pi} \tilde{a}(s+t, \rho(s) \cdot(x, \xi)) d s=0
$$

for all $t$, i.e. coming back to the definition of $\tilde{a}$ and $M_{0}(a)$,

$$
\int_{0}^{2 \pi} a(s+t, \rho(s) \cdot(x, \xi)) d s=\frac{1}{2 \pi} \int_{0}^{2 \pi} \int_{0}^{2 \pi} a\left(s^{\prime}+s+t, \rho\left(s^{\prime}\right) \rho(s) \cdot(x, \xi)\right) d s^{\prime} d s
$$

which follows from the group property of $\rho(\cdot)$. This concludes the proof.

\subsection{Conjugation to an autonomous ODE}

Definition 2.2.1 Let $\ell \in \mathbb{R}_{+}$. One denotes by $\mathcal{P}^{-\ell}$ the space of maps $\Omega$ defined on $\bigcup_{m} \mathcal{S}^{m}\left(\mathbb{S}^{1} \times\right.$ $\left.\mathbb{R}^{d} \times \mathbb{R}^{d}\right)$, with values in the same space, satisfying the following: for any $N \in \mathbb{N}$, there is a family $\left(\Omega_{j}^{N}\right)_{1 \leq j \leq k_{N}}$ of $j$-linear maps

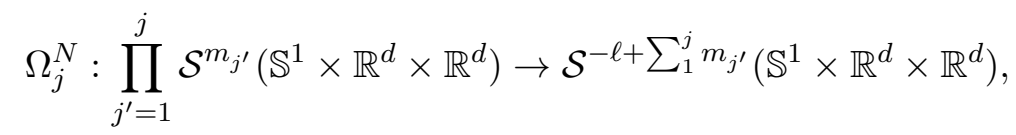

such that if $\Omega^{N}(a)=\sum_{j=1}^{k_{N}} \Omega_{j}^{N}(a, \ldots, a)$, for any $a \in \mathcal{S}^{0}\left(\mathbb{S}^{1} \times \mathbb{R}^{d} \times \mathbb{R}^{d}\right)$

$$
\Omega(a)-\Omega^{N}(a) \in \mathcal{S}^{-2 N-\ell}\left(\mathbb{S}^{1} \times \mathbb{R}^{d} \times \mathbb{R}^{d}\right) .
$$

Remark: It follows from the definition and from (1.2.2), (1.2.3), that if $\Omega^{(1)} \in \mathcal{P}^{-\ell_{1}}, \Omega^{(2)} \in$ $\mathcal{P}^{-\ell_{2}}$, then for any $a \in \mathcal{S}^{0}\left(\mathbb{S}^{1} \times \mathbb{R}^{d} \times \mathbb{R}^{d}\right)$, Op ${ }^{\mathrm{W}}\left(\Omega^{(1)}(a)\right) \circ \mathrm{Op}^{\mathrm{W}}\left(\Omega^{(2)}(a)\right)$ may be written as $\mathrm{Op}^{\mathrm{W}}(\Omega(a))$ for some $\Omega \in \mathcal{P}^{-\left(\ell_{1}+\ell_{2}\right)}$.

Proposition 2.2.2 Let $N \in \mathbb{N}^{*}$. There is a map $\Omega$ belonging to the class $\mathcal{P}^{-2}$ such that the following holds: for any real valued symbol $V$ in $\mathcal{S}^{0}\left(\mathbb{S}^{1} \times \mathbb{R}^{d} \times \mathbb{R}^{d}\right)$, there are symbols $U$ in $\mathcal{S}^{-2 N}\left(\mathbb{S}^{1} \times \mathbb{R}^{d} \times \mathbb{R}^{d}\right), S$ in $\mathcal{S}^{-2 N-2}\left(\mathbb{S}^{1} \times \mathbb{R}^{d} \times \mathbb{R}^{d}\right), m$ in $\mathcal{S}^{0}\left(\mathbb{S}^{1} \times \mathbb{R}^{d} \times \mathbb{R}^{d}\right)$ and a symbol of $\mathcal{S}^{0}\left(\mathbb{R}^{d} \times \mathbb{R}^{d}\right)$, independent of time,

$$
W=\left.\left[M_{0}(V)+\Omega(V)\right]\right|_{t=0},
$$

such that

$$
\begin{aligned}
e^{-i t P_{0}} \mathrm{Op}^{\mathrm{W}}(m)^{*}\left[i \partial_{t}+P_{0}\right. & \left.+\mathrm{Op}^{\mathrm{W}}(V)\right] \mathrm{Op}^{\mathrm{W}}(m) e^{i t P_{0}} \\
= & i \partial_{t}+\mathrm{Op}^{\mathrm{W}}(W)+e^{-i t P_{0}} \mathrm{Op}^{\mathrm{W}}(U) e^{i t P_{0}} \\
& +e^{-i t P_{0}} \mathrm{Op}^{\mathrm{W}}(S) e^{i t P_{0}} \circ\left(i \partial_{t}\right)+\left(i \partial_{t}\right) \circ e^{-i t P_{0}} \mathrm{Op}^{\mathrm{W}}(S) e^{i t P_{0}} .
\end{aligned}
$$

Moreover $W, U, S$ are real valued and

$$
\mathrm{Op}^{\mathrm{W}}(m)^{*} \mathrm{Op}^{\mathrm{W}}(m)-I d=\mathrm{Op}^{\mathrm{W}}(r) \text { with } r \in \mathcal{S}^{-4}\left(\mathbb{S}^{1} \times \mathbb{R}^{d} \times \mathbb{R}^{d}\right) \text {. }
$$


We shall prove the proposition in several steps. We first construct a conjugation allowing one to replace the given symbol of order zero $V$, by the sum of an element of $\mathcal{S}_{Z}^{0}\left(\mathbb{S}^{1} \times \mathbb{R}^{d} \times \mathbb{R}^{d}\right)$ and of a symbol of lower order.

Lemma 2.2.3 Let $V \in \mathcal{S}^{0}\left(\mathbb{S}^{1} \times \mathbb{R}^{d} \times \mathbb{R}^{d}\right)$, real valued. One may find a symbol $q \in \mathcal{S}^{0}\left(\mathbb{S}^{1} \times \mathbb{R}^{d} \times \mathbb{R}^{d}\right)$ satisfying

$$
|q|^{2}=1,\{q, \bar{q}\}=0,
$$

and real valued symbols $V_{0}^{Z}=M_{0}(V) \in \mathcal{S}_{Z}^{0}\left(\mathbb{S}^{1} \times \mathbb{R}^{d} \times \mathbb{R}^{d}\right), V_{1} \in \mathcal{S}^{-2}\left(\mathbb{S}^{1} \times \mathbb{R}^{d} \times \mathbb{R}^{d}\right), S_{1} \in$ $\mathcal{S}^{-4}\left(\mathbb{S}^{1} \times \mathbb{R}^{d} \times \mathbb{R}^{d}\right)$, such that

$$
\begin{aligned}
\mathrm{Op}^{\mathrm{W}}(q)^{*}\left(i \partial_{t}+P_{0}+\mathrm{Op}^{\mathrm{W}}(V)\right) \mathrm{Op}^{\mathrm{W}}(q)= & i \partial_{t}+P_{0}+\mathrm{Op}^{\mathrm{W}}\left(V_{0}^{Z}\right)+\mathrm{Op}^{\mathrm{W}}\left(V_{1}\right) \\
& +\mathrm{Op}^{\mathrm{W}}\left(S_{1}\right)\left(i \partial_{t}+P_{0}\right)+\left(i \partial_{t}+P_{0}\right) \mathrm{Op}^{\mathrm{W}}\left(S_{1}\right) .
\end{aligned}
$$

Moreover $V_{1}$ (resp. $S_{1}$ ) may be written as $\Omega^{(-2)}(V)$ (resp. $\Omega^{(-4)}(V)$ ) for some $\Omega^{(-\ell)} \in \mathcal{P}^{-\ell}$.

Proof: Define $V_{0}^{Z}=M_{0}(V), V_{0}^{B}=V-V_{0}^{Z}$ and take, according to proposition 2.1.2, an element $b \in \mathcal{S}^{0}\left(\mathbb{S}^{1} \times \mathbb{R}^{d} \times \mathbb{R}^{d}\right)$, real valued, solving the equation $\partial_{t} b+\left\{p_{0}, b\right\}=V_{0}^{B}$. Setting $Q=\mathrm{Op}^{\mathrm{W}}(q)$, the left hand side of (2.2.6) may be written

$$
i \partial_{t}+P_{0}+\frac{1}{2}\left[Q^{*} Q-I\right]\left(i \partial_{t}+P_{0}\right)+\frac{1}{2}\left(i \partial_{t}+P_{0}\right)\left[Q^{*} Q-I\right]+\Sigma+Q^{*} \mathrm{Op}^{\mathrm{W}}\left(V_{0}^{Z}\right) Q,
$$

where

$$
\Sigma=\frac{1}{2} Q^{*}\left(\left[i \partial_{t}+P_{0}, Q\right]+\mathrm{Op}^{\mathrm{W}}\left(V_{0}^{B}\right) Q\right)+\frac{1}{2}\left(\left[Q^{*}, i \partial_{t}+P_{0}\right]+Q^{*} \mathrm{Op}^{\mathrm{W}}\left(V_{0}^{B}\right)\right) Q .
$$

We want to choose $Q$ so that $\left[i \partial_{t}+P_{0}, Q\right]+\mathrm{Op}^{\mathrm{W}}\left(V_{0}^{B}\right) Q$ be of order -2 . By (1.2.6) and symbolic calculus, it is enough to solve

$$
i \partial_{t} q+i\left\{p_{0}, q\right\}+V_{0}^{B} q=0 .
$$

We take $q=e^{i b}$. Since $b$ is of order zero and real valued, we get an element of $\mathcal{S}^{0}\left(\mathbb{S}^{1} \times \mathbb{R}^{d} \times \mathbb{R}^{d}\right)$ satisfying $|q|=1$. Moreover, $\{q, \bar{q}\}=0$. By (1.2.2) and (1.2.3), it follows that $\Sigma$ may be written as $\mathrm{Op}^{\mathrm{W}}(\sigma)$ for some symbol $\sigma$ in $\mathcal{S}^{-2}\left(\mathbb{S}^{1} \times \mathbb{R}^{d} \times \mathbb{R}^{d}\right)$, which is real valued since $\Sigma$ is self-adjoint. Consequently, $\Sigma$ contributes to $\mathrm{Op}^{\mathrm{W}}\left(V_{1}\right)$ in the right hand side of (2.2.6).

Since $Q^{*}=\mathrm{Op}^{\mathrm{W}}(\bar{q})$ and since $q \bar{q}=1,\{q, \bar{q}\}=0$, it follows from (1.2.2) and the fact that in (1.2.3) $c_{1}=\frac{i}{2}\{a, b\}$, that $\frac{1}{2}\left(Q^{*} Q-I\right)$ may be written as $\mathrm{Op}^{\mathrm{W}}\left(S_{1}\right)$ for some real valued symbol $S_{1}$ in $\mathcal{S}^{-4}\left(\mathbb{S}^{1} \times \mathbb{R}^{d} \times \mathbb{R}^{d}\right)$. Let us show that the last term in $(2.2 .7), Q^{*} \mathrm{Op}^{\mathrm{W}}\left(V_{0}^{Z}\right) Q$, may be written as $\mathrm{Op}^{\mathrm{W}}\left(V_{0}^{Z}\right)+\mathrm{Op}^{\mathrm{W}}(\sigma)$, where $\sigma$ will be again a real valued element of $\mathcal{S}^{-2}\left(\mathbb{S}^{1} \times \mathbb{R}^{d} \times \mathbb{R}^{d}\right)$ that will contribute to $V_{1}$ in (2.2.6). Actually, this follows from (1.2.4) and the fact that $Q^{*} Q-I$ is of order -4 .

To conclude the proof, we still have to show that $V_{1}$ (resp. $S_{1}$ ) may be written as $\Omega^{(-2)}(V)$ (resp. $\Omega^{(-4)}(V)$ ) for some $\Omega^{(-\ell)} \in \mathcal{P}^{-\ell}$. Notice first that $b$, given by (2.1.5) with $a$ replaced by $V$, is linear in $V$. Moreover, it follows from $(1.2 .2),(1.2 .3)$ that if $a$ is a symbol, $\mathrm{Op}^{\mathrm{W}}(a) \circ \mathrm{Op}^{\mathrm{W}}(q)$ 
may be written as $\mathrm{Op}^{\mathrm{W}}\left(e^{i b} c\right)$, where $c$ has an expansion $\sum c_{k}$, in which each $c_{k}$ is a finite sum of expressions that are linear in $a$ and multi-linear in $b$ (and their derivatives). Applying this to $a=\bar{q}$, we see that $\frac{1}{2}\left[\mathrm{Op}^{\mathrm{W}}(q)^{*} \mathrm{Op}^{\mathrm{W}}(q)-I\right]=\mathrm{Op}^{\mathrm{W}}\left(S_{1}\right)$ is given by a symbol $S_{1}$, such that each term in its expansion is a finite sum of multi-linear expressions in $V$ and its derivatives. Since $S_{1}$ is of order -4 , this means that $S_{1}$ may be written as $\Omega^{(-4)}(V)$ for $\Omega^{(-4)} \in \mathcal{P}^{-4}$. In the same way, the contributions to $V_{1}$ coming from $\Sigma$, or from the symbol of $Q^{*} \mathrm{Op}^{\mathrm{W}}\left(V_{0}^{Z}\right) Q$, may be written as $\Omega^{(-2)}(V)$ for some $\Omega^{(-2)} \in \mathcal{P}^{-2}$. This concludes the proof.

The second step of the proof of proposition 2.2.2 is to conjugate the right hand side of (2.2.6) to an expression where $V_{1}$ and $S_{1}$ will be replaced by symbols of arbitrary negative order. We do that by induction. Next lemma is the general step.

Lemma 2.2.4 Let $j$ be a positive integer. Assume given real valued symbols $V_{j-1}^{Z}$ in $\mathcal{S}_{Z}^{0}\left(\mathbb{S}^{1} \times\right.$ $\left.\mathbb{R}^{d} \times \mathbb{R}^{d}\right), V_{j}$ in $\mathcal{S}^{-2 j}\left(\mathbb{S}^{1} \times \mathbb{R}^{d} \times \mathbb{R}^{d}\right), S_{j}$ in $\mathcal{S}^{-2 j-2}\left(\mathbb{S}^{1} \times \mathbb{R}^{d} \times \mathbb{R}^{d}\right)$, a in $\mathcal{S}^{-2 j}\left(\mathbb{S}^{1} \times \mathbb{R}^{d} \times \mathbb{R}^{d}\right)$, $a^{\prime \prime}$ in $\mathcal{S}^{-2 j-2}\left(\mathbb{S}^{1} \times \mathbb{R}^{d} \times \mathbb{R}^{d}\right)$. One may find real valued symbols $\widetilde{V}_{j+1} \in \mathcal{S}^{-2 j-2}\left(\mathbb{S}^{1} \times \mathbb{R}^{d} \times \mathbb{R}^{d}\right)$, $\widetilde{S}_{j+1} \in \mathcal{S}^{-2 j-4}\left(\mathbb{S}^{1} \times \mathbb{R}^{d} \times \mathbb{R}^{d}\right)$ such tat if $a=i a^{\prime}+a^{\prime \prime}$, the operator

$$
\begin{aligned}
\left(I+\mathrm{Op}^{\mathrm{W}}(a)\right)^{*}[ & i \partial_{t}+P_{0}+\mathrm{Op}^{\mathrm{W}}\left(V_{j-1}^{Z}\right)+\mathrm{Op}^{\mathrm{W}}\left(V_{j}\right) \\
& \left.+\mathrm{Op}^{\mathrm{W}}\left(S_{j}\right)\left(i \partial_{t}+P_{0}\right)+\left(i \partial_{t}+P_{0}\right) \mathrm{Op}^{\mathrm{W}}\left(S_{j}\right)\right]\left(I+\mathrm{Op}^{\mathrm{W}}(a)\right)
\end{aligned}
$$

may be written as

$$
\begin{aligned}
& i \partial_{t}+P_{0}+\mathrm{Op}^{\mathrm{W}}\left(V_{j-1}^{Z}\right)+\mathrm{Op}^{\mathrm{W}}\left(V_{j}\right)-i\left[\mathrm{Op}^{\mathrm{W}}\left(a^{\prime}\right), i \partial_{t}+P_{0}\right] \\
& \left.+\mathrm{Op}^{\mathrm{W}}\left(a^{\prime \prime}\right)+\mathrm{Op}^{\mathrm{W}}\left(S_{j}\right)+\frac{1}{2} \mathrm{Op}^{\mathrm{W}}\left(a^{\prime}\right)^{*} \mathrm{Op}^{\mathrm{W}}\left(a^{\prime}\right)\right]\left(i \partial_{t}+P_{0}\right) \\
& +\left(i \partial_{t}+P_{0}\right)\left[\mathrm{Op}^{\mathrm{W}}\left(a^{\prime \prime}\right)+\mathrm{Op}^{\mathrm{W}}\left(S_{j}\right)+\frac{1}{2} \mathrm{Op}^{\mathrm{W}}\left(a^{\prime}\right)^{*} \mathrm{Op}^{\mathrm{W}}\left(a^{\prime}\right)\right] \\
& +\mathrm{Op}^{\mathrm{W}}\left(\widetilde{V}_{j+1}\right)+\mathrm{Op}^{\mathrm{W}}\left(\widetilde{S}_{j+1}\right)\left(i \partial_{t}+P_{0}\right)+\left(i \partial_{t}+P_{0}\right) \mathrm{Op}^{\mathrm{W}}\left(\widetilde{S}_{j+1}\right) .
\end{aligned}
$$

Moreover, if we assume that $V_{j-1}^{Z}$ (resp. $V_{j}$, resp. $S_{j}$, resp. $a^{\prime}$, resp. $\left.a^{\prime \prime}\right)$ may be written in terms of a given symbol of order zero $V$ as $\Omega^{(0)}(V)$ (resp. $\Omega^{(-2 j)}(V)$, resp. $\Omega^{(-2 j-2)}(V)$, resp. $\Omega^{(-2 j)}(V)$, resp. $\left.\Omega^{(-2 j-2)}(V)\right)$ for elements $\Omega^{(-\ell)} \in \mathcal{P}^{-\ell}$, then $\widetilde{V}_{j+1}$ (resp. $\widetilde{S}_{j+1}$ ) is of the form $\Omega^{(-2 j-2)}(V)$ (resp. $\Omega^{(-2 j-4)}(V)$ ) for other elements $\Omega^{(-\ell)}$ of $\mathcal{P}^{-\ell}$.

Proof: Since $\mathrm{Op}^{\mathrm{W}}\left(a^{\prime}\right), \mathrm{Op}^{\mathrm{W}}\left(a^{\prime \prime}\right), \mathrm{Op}^{\mathrm{W}}\left(S_{j}\right)$ are self-adjoint, we may write (2.2.9) as

$$
\begin{aligned}
& i \partial_{t}+P_{0}+\mathrm{Op}^{\mathrm{W}}\left(V_{j-1}^{Z}\right)+\mathrm{Op}^{\mathrm{W}}\left(V_{j}\right)-i\left[\mathrm{Op}^{\mathrm{W}}\left(a^{\prime}\right), i \partial_{t}+P_{0}\right] \\
& \left.+\mathrm{Op}^{\mathrm{W}}\left(a^{\prime \prime}\right)+\mathrm{Op}^{\mathrm{W}}\left(S_{j}\right)\right)\left(i \partial_{t}+P_{0}\right)+\left(i \partial_{t}+P_{0}\right)\left(\mathrm{Op}^{\mathrm{W}}\left(a^{\prime \prime}\right)+\mathrm{Op}^{\mathrm{W}}\left(S_{j}\right)\right) \\
& -i\left[\mathrm{Op}^{\mathrm{W}}\left(a^{\prime}\right), \mathrm{Op}^{\mathrm{W}}\left(V_{j-1}^{Z}\right)\right]+\mathrm{Op}^{\mathrm{W}}\left(a^{\prime \prime}\right) \mathrm{Op}^{\mathrm{W}}\left(V_{j-1}^{Z}\right)+\mathrm{Op}^{\mathrm{W}}\left(V_{j-1}^{Z}\right) \mathrm{Op}^{\mathrm{W}}\left(a^{\prime \prime}\right) \\
& +\mathrm{Op}^{\mathrm{W}}(a)^{*} \mathrm{Op}^{\mathrm{W}}\left(V_{j}\right)+\mathrm{Op}^{\mathrm{W}}\left(V_{j}\right) \mathrm{Op}^{\mathrm{W}}(a) \\
& +\mathrm{Op}^{\mathrm{W}}(a)^{*} \mathrm{Op}^{\mathrm{W}}\left(S_{j}\right)^{*}\left(i \partial_{t}+P_{0}\right)+\left(i \partial_{t}+P_{0}\right) \mathrm{Op}^{\mathrm{W}}\left(S_{j}\right) \mathrm{Op}^{\mathrm{W}}(a) \\
& +\mathrm{Op}^{\mathrm{W}}(a)^{*}\left(i \partial_{t}+P_{0}\right) \mathrm{Op}^{\mathrm{W}}(a) \\
& +\mathrm{Op}^{\mathrm{W}}(a)^{*} \mathrm{Op}^{\mathrm{W}}\left(V_{j-1}^{Z}+V_{j}\right) \mathrm{Op}^{\mathrm{W}}(a) \\
& +\mathrm{Op}^{\mathrm{W}}(a)^{*} \mathrm{Op}^{\mathrm{W}}\left(S_{j}\right)^{*}\left(i \partial_{t}+P_{0}\right) \mathrm{Op}^{\mathrm{W}}(a)+\mathrm{Op}^{\mathrm{W}}(a)^{*}\left(i \partial_{t}+P_{0}\right) \mathrm{Op}^{\mathrm{W}}\left(S_{j}\right) \mathrm{Op}^{\mathrm{W}}(a) .
\end{aligned}
$$


The first two lines of (2.2.11) give contributions to the first three lines of (2.2.10). Consider the sixth line in (2.2.11). Write

$$
\begin{aligned}
\mathrm{Op}^{\mathrm{W}}(a)^{*}\left(i \partial_{t}+P_{0}\right) \mathrm{Op}^{\mathrm{W}}(a)= & \frac{1}{2}\left[\mathrm{Op}^{\mathrm{W}}(a)^{*} \mathrm{Op}^{\mathrm{W}}(a)\left(i \partial_{t}+P_{0}\right)+\left(i \partial_{t}+P_{0}\right) \mathrm{Op}^{\mathrm{W}}(a)^{*} \mathrm{Op}^{\mathrm{W}}(a)\right] \\
& +\frac{1}{2}\left[\mathrm{Op}^{\mathrm{W}}(a)^{*}, i \partial_{t}+P_{0}\right] \mathrm{Op}^{\mathrm{W}}(a)+\frac{1}{2} \mathrm{Op}^{\mathrm{W}}(a)^{*}\left[i \partial_{t}+P_{0}, \mathrm{Op}^{\mathrm{W}}(a)\right] .
\end{aligned}
$$

Since $a$ is of order $-2 j,(1.2 .2)$ and (1.2.4) show that the last two terms are of order $-4 j \leq$ $-2 j-2$, so contribute to the $\widetilde{V}_{j+1}$ term in (2.2.10). On the other hand

$$
\mathrm{Op}^{\mathrm{W}}(a)^{*} \mathrm{Op}^{\mathrm{W}}(a)\left(i \partial_{t}+P_{0}\right)=\mathrm{Op}^{\mathrm{W}}\left(a^{\prime}\right)^{*} \mathrm{Op}^{\mathrm{W}}\left(a^{\prime}\right)\left(i \partial_{t}+P_{0}\right)+\mathrm{Op}^{\mathrm{W}}(b)\left(i \partial_{t}+P_{0}\right)
$$

for some symbol $b$ of order $-4 j-2 \leq-2 j-2$, again by (1.2.2). The first term in the right hand side gives the missing contribution in the second line of (2.2.10). The second term contributes to the $S_{j+1}$ term in (2.2.10). These contributions are real valued by self-adjointness.

By (1.2.2) and (1.2.4), the third and fourth lines of (2.2.11) are made of self-adjoint operators of order $-2 j-2$, so will contribute to the $\mathrm{Op}^{\mathrm{W}}\left(\widetilde{V}_{j+1}\right)$ term in $(2.2 .10)$. In the fifth line, write

$$
\mathrm{Op}^{\mathrm{W}}\left(S_{j}\right) \mathrm{Op}^{\mathrm{W}}(a)=\mathrm{Op}^{\mathrm{W}}\left(c_{1}\right)+\mathrm{Op}^{\mathrm{W}}\left(c_{2}\right)
$$

where $c_{1}, c_{2}$ are of order $-4 j-2 \leq-2 j-4, c_{1}$ real, $c_{2}$ purely imaginary. The fifth line of $(2.2 .11)$ is thus

$$
\mathrm{Op}^{\mathrm{W}}\left(c_{1}\right)\left(i \partial_{t}+P_{0}\right)+\left(i \partial_{t}+P_{0}\right) \mathrm{Op}^{\mathrm{W}}\left(c_{1}\right)+\left[i \partial_{t}+P_{0}, \mathrm{Op}^{\mathrm{W}}\left(c_{2}\right)\right] .
$$

The last term contributes to $\mathrm{Op}^{\mathrm{W}}\left(\widetilde{V}_{j+1}\right)$ and the first two ones to the last two expressions in (2.2.10).

The last but one line in (2.2.11) is of order $-4 j \leq-2 j-2$ and self-adjoint, so contributes to $\mathrm{Op}^{\mathrm{W}}\left(\widetilde{V}_{j+1}\right)$ in $(2.2 \cdot 10)$.

Consider finally the first term in the last line of (2.2.11) and write it as

$$
\mathrm{Op}^{\mathrm{W}}(a)^{*} \mathrm{Op}^{\mathrm{W}}\left(S_{j}\right)^{*} \mathrm{Op}^{\mathrm{W}}(a)\left(i \partial_{t}+P_{0}\right)+\mathrm{Op}^{\mathrm{W}}(a)^{*} \mathrm{Op}^{\mathrm{W}}\left(S_{j}\right)^{*}\left[i \partial_{t}+P_{0}, \mathrm{Op}^{\mathrm{W}}(a)\right] .
$$

Again, by symbolic calculus, the last term is of order $-6 j-2 \leq-2 j-2$, so contributes to $\mathrm{Op}^{\mathrm{W}}\left(\widetilde{V}_{j+1}\right)$ in $(2.2 .10)$. The coefficient of $\left(i \partial_{t}+P_{0}\right)$ in the first term is also of order $-6 j-2 \leq$ $-2 j-4$, so gives rise to a contribution to the last but one term in (2.2.10). These contributions are real by self-adjointness. The assertions of the end of the statement of the lemma, concerning the structure of $\widetilde{V}_{j+1}, \widetilde{S}_{j+1}$ as expressions of form $\Omega^{(-2 j-2)}(V), \Omega^{(-2 j-4)}(V)$, follow from the fact that the corresponding operators are obtained by composition from operators of the same form, using the remark following definition 2.2.1.

The last but one step in the proof of proposition 2.2.2 is the following:

Lemma 2.2.5 Let $V$ be a real valued symbol in $\mathcal{S}^{0}\left(\mathbb{S}^{1} \times \mathbb{R}^{d} \times \mathbb{R}^{d}\right)$ and $N$ a positive integer. One may find a real symbol $V^{Z}$ in $\mathcal{S}_{Z}^{0}\left(\mathbb{S}^{1} \times \mathbb{R}^{d} \times \mathbb{R}^{d}\right)$, a real symbol $V_{N+1}$ in $\mathcal{S}^{-2 N-2}\left(\mathbb{S}^{1} \times \mathbb{R}^{d} \times \mathbb{R}^{d}\right)$, a real symbol $S_{N+1}$ in $\mathcal{S}^{-2 N-4}\left(\mathbb{S}^{1} \times \mathbb{R}^{d} \times \mathbb{R}^{d}\right)$, a symbol $m \in \mathcal{S}^{0}\left(\mathbb{S}^{1} \times \mathbb{R}^{d} \times \mathbb{R}^{d}\right)$, such that

$$
\mathrm{Op}^{\mathrm{W}}(m) \mathrm{Op}^{\mathrm{W}}(m)^{*}-I=\mathrm{Op}^{\mathrm{W}}(r) \text { with } r \in \mathcal{S}^{-4}\left(\mathbb{S}^{1} \times \mathbb{R}^{d} \times \mathbb{R}^{d}\right),
$$


and

$$
\begin{array}{r}
\mathrm{Op}^{\mathrm{W}}(m)^{*}\left(i \partial_{t}+P_{0}+\mathrm{Op}^{\mathrm{W}}(V)\right) \mathrm{Op}^{\mathrm{W}}(m)=i \partial_{t}+P_{0}+\mathrm{Op}^{\mathrm{W}}\left(V^{Z}\right)+\mathrm{Op}^{\mathrm{W}}\left(V_{N+1}\right) \\
+\mathrm{Op}^{\mathrm{W}}\left(S_{N+1}\right)\left(i \partial_{t}+P_{0}\right)+\left(i \partial_{t}+P_{0}\right) \mathrm{Op}^{\mathrm{W}}\left(S_{N+1}\right) .
\end{array}
$$

Moreover we may write

$$
V^{Z}=M_{0}(V)+\Omega^{(-2)}(V),
$$

where $M_{0}$ is given by (2.1.3) and $\Omega^{(-2)}$ is an element of $\mathcal{P}^{-2}$.

Proof: We proceed by induction on $N$. By lemma 2.2.3, formula (2.2.13) holds with $N=$ $0, m=q$. Assume that (2.2.13) has been proved at some order $N=j-1$, with $V^{Z}$ in the right hand side replaced by some $V_{j-1}^{Z}$, and with a symbol $V_{j}$ (resp. $S_{j}$ ) that may be written as $\Omega^{(-2 j)}(V)$ (resp. $\Omega^{(-2 j-2)}(V)$ ) for some $\Omega^{(-2 j)}$ in $\mathcal{P}^{-2 j}$ (resp. $\Omega^{(-2 j-2)}$ in $\mathcal{P}^{-(2 j+2)}$ ). We compose this equality on the left with $\left(I+\mathrm{Op}^{\mathrm{W}}(a)\right)$, for some symbol $a=i a^{\prime}+a^{\prime \prime}$ to be chosen, with $a^{\prime}$ in $\mathcal{S}^{-2 j}\left(\mathbb{S}^{1} \times \mathbb{R}^{d} \times \mathbb{R}^{d}\right)$, $a^{\prime \prime}$ in $\mathcal{S}^{-2 j-2}\left(\mathbb{S}^{1} \times \mathbb{R}^{d} \times \mathbb{R}^{d}\right)$, $a^{\prime}$ and $a^{\prime \prime}$ real valued. We apply lemma 2.2.4 to get expressions (2.2.10), in which we decompose $V_{j}=M_{0}\left(V_{j}\right)+V_{j}^{B}$, where $M_{0}\left(V_{j}\right) \in \mathcal{S}_{Z}^{-2 j}\left(\mathbb{S}^{1} \times \mathbb{R}^{d} \times \mathbb{R}^{d}\right), V_{j}^{B} \in \mathcal{S}_{B}^{-2 j}\left(\mathbb{S}^{1} \times \mathbb{R}^{d} \times \mathbb{R}^{d}\right)$. We define $V_{j}^{Z}=V_{j-1}^{Z}+M_{0}\left(V_{j}\right)$. Since $V_{j}$ may be written $\Omega^{(-2 j)}(V)$, we obtain from assumption (2.2.14) for $V_{j-1}^{Z}$ that the same type of equality will be satisfied by $V_{j}^{Z}$. We choose next $a^{\prime}$ so that

$$
-i\left[\mathrm{Op}^{\mathrm{W}}\left(a^{\prime}\right), i \partial_{t}+P_{0}\right]+\mathrm{Op}^{\mathrm{W}}\left(V_{j}^{B}\right)
$$

is an operator of order $-2 j-2$. At the level of symbols, we have to solve $\partial_{t} a^{\prime}+\left\{p_{0}, a^{\prime}\right\}=V_{j}^{B}$, which is possible by proposition 2.1.2. We get a real symbol $a^{\prime}$ of order $-2 j$ given by a linear expression in $V_{j}^{B}$, so that may be written as $\Omega^{(-2 j)}(V)$ for some $\Omega^{(-2 j)}$ in $\mathcal{P}^{-2 j}$. In that way, (2.2.15) is an operator of order $-2 j-2$, self-adjoint, which contributes to $V_{j+1}$ in (2.2.13) at rank $N=j$. The $\widetilde{V}_{j+1}$ contribution to (2.2.10) may be as well incorporated inside $V_{j+1}$. We choose next

$$
a^{\prime \prime}=-S_{j}-\frac{1}{2}\left|a^{\prime}\right|^{2} \in \mathcal{S}^{-2 j-2}\left(\mathbb{S}^{1} \times \mathbb{R}^{d} \times \mathbb{R}^{d}\right) .
$$

By the induction hypothesis, $a^{\prime \prime}$ may be written $\Omega^{(-2 j-2)}(V)$ for some $\Omega^{(-2 j-2)}$ in $\mathcal{P}^{-(2 j+2)}$, and this definition of $a^{\prime \prime}$ implies that the coefficient of $\left(i \partial_{t}+P_{0}\right)$, in the second and third lines of (2.2.10), may be written $\mathrm{Op}^{\mathrm{W}}(c)$ for a real symbol $c$ in $\mathcal{S}^{-2 j-4}\left(\mathbb{S}^{1} \times \mathbb{R}^{d} \times \mathbb{R}^{d}\right)$. Moreover, $c$ may be written as $\Omega^{(-2 j-4)}(V)$ for some $\Omega^{(-2 j-4)}$ in $\mathcal{P}^{-2(j+2)}$. This symbol, together with $\widetilde{S}_{j+1}$ in (2.2.10), contributes to $S_{j+1}$ in (2.2.13) at rank $N=j$. Since $(2.2 .12)$, with $\mathrm{Op}^{\mathrm{W}}(m)$ replaced by $\mathrm{Op}^{\mathrm{W}}(m)\left(I+\mathrm{Op}^{\mathrm{W}}(a)\right)$, holds as

$$
\left(I+\mathrm{Op}^{\mathrm{W}}(a)\right)^{*}\left(I+\mathrm{Op}^{\mathrm{W}}(a)\right)-I=2 \mathrm{Op}^{\mathrm{W}}\left(a^{\prime \prime}\right)+\mathrm{Op}^{\mathrm{W}}(a)^{*} \mathrm{Op}^{\mathrm{W}}(a)
$$

is of order $-2 j-2 \leq-4$, we have obtained (2.2.12) and (2.2.13) at rank $j$. This concludes the proof of the lemma.

Proof of proposition 2.2.2: We multiply (2.2.13) with $N$ replaced by $N-1$ by $e^{-i t P_{0}}$ on the left and $e^{i t P_{0}}$ on the right. The main contribution of the right hand side of (2.2.13) is

$$
e^{-i t P_{0}}\left(i \partial_{t}+P_{0}+\mathrm{Op}^{\mathrm{W}}\left(V^{Z}\right)\right) e^{i t P_{0}}=i \partial_{t}+e^{-i t P_{0}} \mathrm{Op}^{\mathrm{W}}\left(V^{Z}\right) e^{i t P_{0}} .
$$


Notice that

$$
\frac{d}{d t}\left[e^{-i t P_{0}} \mathrm{Op}^{\mathrm{W}}\left(V^{Z}\right) e^{i t P_{0}}\right]=e^{-i t P_{0}}\left[\mathrm{Op}^{\mathrm{W}}\left(\partial_{t} V^{Z}\right)+i\left[\mathrm{Op}^{\mathrm{W}}\left(V^{Z}\right), P_{0}\right]\right] e^{i t P_{0}} .
$$

By the second formula (1.2.6) and the fact that $V^{Z}$ is in $\mathcal{S}_{Z}^{0}\left(\mathbb{S}^{1} \times \mathbb{R}^{d} \times \mathbb{R}^{d}\right)$, we see that this quantity vanishes. Consequently, (2.2.16) is equal to $\left.i \partial_{t}+\left.\mathrm{Op}^{\mathrm{W}}\left(V^{Z}\right)\right|_{t=0}\right)$. We define $W(x, \xi)=$ $V^{Z}(0, x, \xi)$. This is a symbol of order 0 , independent of time, and (2.2.14) gives (2.2.2). The contributions coming by conjugation from the $V_{N}$ and $S_{N}$ terms of the right hand side of (2.2.13) give the last three terms of (2.2.3). This concludes the proof.

\section{Construction of the solution}

This section is devoted to the construction of a potential $V$ and of a solution $u$ to (1.1.5) for which (1.1.6) holds. The main step will be to show that, if $W$ is a convenient autonomous symbol belonging to $\mathcal{S}^{0}(\mathbb{R} \times \mathbb{R})$, we may construct an approximate solution of the equation $\left(i \partial_{t}+\mathrm{Op}^{\mathrm{W}}(W)\right) u=0$ satisfying (1.1.6). Then, inverting (2.2.2), we shall obtain a time dependent potential $V$, such that the corresponding Schrödinger equation $i \partial_{t}+P_{0}+\mathrm{Op}^{\mathrm{W}}(V)$ may be reduced by conjugation to the right hand side of (2.2.3). This right hand side being equal to $\left(i \partial_{t}+\mathrm{Op}^{\mathrm{W}}(W)\right)$ modulo operators of very negative order, we shall deduce from the approximate solution already constructed an approximate solution of $\left(i \partial_{t}+P_{0}+\mathrm{Op}^{\mathrm{W}}(V)\right) u=0$. Modifying it to get an exact solution, we shall obtain the conclusion of theorem 1.1.3.

We first study the Hamiltonian flow of an autonomous symbol of order zero satisfying convenient assumptions. From now on, the space dimension is $d=1$.

\subsection{The Hamiltonian flow associated to a potential}

We use polar coordinates $(\rho, \theta)$ on $T^{*} \mathbb{R}(x=\rho \cos \theta, \xi=\rho \sin \theta)$. A function $W$ on $T^{*} \mathbb{R}$ will be denoted indifferently by $W(x, \xi)$ or $W(\rho, \theta)$. Let $W \in \mathcal{S}^{0}(\mathbb{R} \times \mathbb{R})$ satisfy the following assumptions: There is $m>2$, a symbol $\widetilde{W} \in \mathcal{S}^{-m}(\mathbb{R} \times \mathbb{R})$ and a symbol $W_{\infty}$ in $\mathcal{S}^{0}(\mathbb{R} \times \mathbb{R})$, with $W_{\infty}(\lambda(x, \xi))=W_{\infty}(x, \xi)$ for any $\lambda \geq 1$, any $(x, \xi)$ with $x^{2}+\xi^{2} \geq 1$, such that $W=W_{\infty}+\widetilde{W}$. In polar coordinates, we may write for $\rho \geq 1$

$$
W(\rho, \theta)=W_{\infty}(\theta)+\widetilde{W}(\rho, \theta),
$$

with uniform estimates for $\rho \geq 1$

$$
\partial_{\theta}^{\alpha} W_{\infty}(\theta)=O(1),\left(\rho \partial_{\rho}\right)^{\alpha} \partial_{\theta}^{\beta} \widetilde{W}(\rho, \theta)=O\left(\rho^{-m}\right) .
$$

For $A$ a large positive constant to be chosen, we consider the Lagrangian submanifold of $T^{*} \mathbb{R}$ given in cartesian and polar coordinates by

$$
\begin{aligned}
\Lambda_{0}^{A} & =\{(A s \cos (s / A), A s \sin (s / A)) ; s \in[1,2]\} \\
& =\left\{\left(\rho_{0}(s), \theta_{0}(s)\right) ; \rho_{0}(s)=A s, \theta_{0}(s)=\frac{s}{A} ; s \in[1,2]\right\} .
\end{aligned}
$$


Our objective is to show that, under convenient assumptions on $W$, and for large enough $A$, the Hamiltonian flow of the symbol, given in cartesian coordinates by

$$
\dot{x}=-\frac{\partial W}{\partial \xi}, \dot{\xi}=\frac{\partial W}{\partial x},
$$

and in polar coordinates by

$$
\dot{\rho}=-\frac{1}{\rho} \frac{\partial W}{\partial \theta}, \dot{\theta}=\frac{1}{\rho} \frac{\partial W}{\partial \rho}
$$

is globally well defined for initial data in $\Lambda_{0}^{A}$, and transports at large time $t>0$ the Lagrangian $\Lambda_{0}^{A}$ onto a Lagrangian $\Lambda_{t}^{A}$, which is the graph of a function.

Proposition 3.1.1 Assume

$$
\frac{\partial W_{\infty}}{\partial \theta}(0)<0, \frac{\partial^{2} W_{\infty}}{\partial \theta^{2}}(0) \neq 0 .
$$

Denote by $(\rho(t, s), \theta(t, s))$ the flow of (3.1.5) at time $t$, issued from the point $\left(\rho_{0}(s), \theta_{0}(s)\right)$ with parameter $s$ on $\Lambda_{0}^{A}$ at time $t=0$. There are $A_{0}>0, C>0$, such that for any $A \geq A_{0}$, any $s \in[1,2]$, the flow is defined for any $t \geq 0$ and satisfies

$$
C^{-1}\left(A^{2}+t\right)^{1 / 2} \leq \rho(t, s) \leq C\left(A^{2}+t\right)^{1 / 2} .
$$

Moreover, there are smooth functions $s \rightarrow \lambda(s), s \rightarrow \theta_{\infty}(s)$, defined on $[1,2]$, satisfying for any $s \in[1,2]$

$$
C^{-1} \leq \lambda(s) \leq C, C^{-1} A^{-1} \leq\left|\frac{\partial \lambda}{\partial s}(s)\right| \leq C A^{-1},\left|\theta_{\infty}(s)-\frac{s}{A}\right| \leq \frac{C}{A^{m}},
$$

and smooth functions $\sigma_{j}:[0,+\infty[\times[1,2] \rightarrow \mathbb{R}$, satisfying

$$
\begin{aligned}
& \left|\partial_{t}^{\alpha} \partial_{s}^{\beta} \sigma_{1}(t, s)\right| \leq C_{\alpha \beta}\left(A^{2}+t\right)^{-1-\alpha} \\
& \left|\partial_{t}^{\alpha} \partial_{s}^{\beta} \sigma_{2}(t, s)\right| \leq C_{\alpha \beta}\left(A^{2}+t\right)^{-\frac{m}{2}-\alpha},
\end{aligned}
$$

such that

$$
\begin{aligned}
& \rho(t, s)=\left(t \lambda(s)+(A s)^{2}\right)^{1 / 2}\left(1+\sigma_{1}(t, s)\right) \\
& \theta(t, s)=\theta_{\infty}(s)+\sigma_{2}(t, s) .
\end{aligned}
$$

Before starting the proof, we introduce some notation. Let us write equation (3.1.5) under an integral form

$$
\begin{aligned}
\rho(t, s)^{2} & =(A s)^{2}-2 \int_{0}^{t} \frac{\partial W}{\partial \theta}\left(\rho\left(t^{\prime}, s\right), \theta\left(t^{\prime}, s\right)\right) d t^{\prime} \\
\theta(t, s) & =\frac{s}{A}+\int_{0}^{t} \frac{1}{\rho\left(t^{\prime}, s\right)} \frac{\partial \widetilde{W}}{\partial \rho}\left(\rho\left(t^{\prime}, s\right), \theta\left(t^{\prime}, s\right)\right) d t^{\prime}
\end{aligned}
$$


so that, in the second equation, the right hand side depends only on $\widetilde{W}$, which is of order $-m$. One sees by induction that there are, for any $j \in \mathbb{N}^{*}$, and for any $(a, b) \in \mathbb{N} \times \mathbb{N}$ with $2 \leq a+b \leq j$,

- symbols $W_{a b}^{\prime}$ (resp. $W_{a b}^{\prime \prime}$ ) of degree $-m-2 a$ when $a>0$ and 0 when $a=0$ (resp. of degree $-m-2-2 a)$,

- polynomials $P_{a b}^{\prime}\left(X_{1}, \ldots, X_{j+1-a-b}, Y_{1}, \ldots, Y_{j+1-a-b}\right)$, which are homogeneous of degree $a$ in $X=\left(X_{1}, \ldots, X_{j+1-a-b}\right)$ and $b$ in $Y=\left(Y_{1}, \ldots, Y_{j+1-a-b}\right)$, such that

$$
\begin{aligned}
\partial_{s}^{j}\left[\rho(t, s)^{2}\right]=\partial_{s}^{j}\left[(A s)^{2}\right] & -\int_{0}^{t} \frac{1}{\rho\left(t^{\prime}, s\right)} \frac{\partial^{2} \widetilde{W}}{\partial \rho \partial \theta}\left(\rho\left(t^{\prime}, s\right), \theta\left(t^{\prime}, s\right)\right) \partial_{s}^{j}\left[\rho\left(t^{\prime}, s\right)^{2}\right] d t^{\prime} \\
& -2 \int_{0}^{t} \frac{\partial^{2} W}{\partial \theta^{2}}\left(\rho\left(t^{\prime}, s\right), \theta\left(t^{\prime}, s\right)\right) \partial_{s}^{j} \theta\left(t^{\prime}, s\right) d t^{\prime} \\
& +\sum_{2 \leq a+b \leq j} \int_{0}^{t} W_{a b}^{\prime}\left(\rho\left(t^{\prime}, s\right), \theta\left(t^{\prime}, s\right)\right) P_{a b}^{\prime}\left(\left(\partial_{s}^{\alpha} \rho^{2}\right)_{\alpha>0},\left(\partial_{s}^{\beta} \theta\right)_{\beta>0}\right) d t^{\prime}
\end{aligned}
$$

and

$$
\begin{aligned}
\partial_{s}^{j}[\theta(t, s)]=\partial_{s}^{j}\left[\frac{s}{A}\right] & +\frac{1}{2} \int_{0}^{t}\left(\frac{1}{\rho} \frac{\partial}{\partial \rho}\left[\frac{1}{\rho} \frac{\partial \widetilde{W}}{\partial \rho}\right]\right)\left(\rho\left(t^{\prime}, s\right), \theta\left(t^{\prime}, s\right)\right) \partial_{s}^{j}\left[\rho\left(t^{\prime}, s\right)^{2}\right] d t^{\prime} \\
& +\int_{0}^{t} \frac{\partial}{\partial \theta}\left[\frac{1}{\rho} \frac{\partial \widetilde{W}}{\partial \rho}\right]\left(\rho\left(t^{\prime}, s\right), \theta\left(t^{\prime}, s\right)\right) \partial_{s}^{j} \theta\left(t^{\prime}, s\right) d t^{\prime} \\
& +\sum_{2 \leq a+b \leq j} \int_{0}^{t} W_{a b}^{\prime \prime}\left(\rho\left(t^{\prime}, s\right), \theta\left(t^{\prime}, s\right)\right) P_{a b}^{\prime \prime}\left(\left(\partial_{s}^{\alpha} \rho^{2}\right)_{\alpha>0},\left(\partial_{s}^{\beta} \theta\right)_{\beta>0}\right) d t^{\prime}
\end{aligned}
$$

In (3.1.12), $a$ (resp. $b$ ) represents the number of times $\frac{\partial W}{\partial \theta}$ has been differentiated by $\frac{1}{\rho} \frac{\partial}{\partial \rho}$ (resp. $\left.\frac{\partial}{\partial \theta}\right)$ to compute $W_{a b}^{\prime}$. In the same way, in (3.1.13), a (resp. b) is the number of $\frac{1}{\rho} \frac{\partial}{\partial \rho}$ (resp. $\frac{\partial}{\partial \theta}$ ) derivatives acting on $\frac{1}{\rho} \frac{\partial \widetilde{W}}{\partial \rho}$ to give $W_{a b}^{\prime \prime}$. The polynomial expressions $P_{a b}^{\prime}, P_{a b}^{\prime \prime}$ give the multi-linear expressions, homogeneous of degree $a$ (resp. $b$ ) in the derivatives of $\rho^{2}$ (resp. $\theta$ ), that multiply these symbols. The conditions on the orders of $W_{a b}^{\prime}, W_{a b}^{\prime \prime}$ come from the fact that $\frac{1}{\rho} \frac{\partial}{\partial \rho}$ (resp. $\frac{\partial}{\partial \theta}$ ) acting on a symbol gives a gain of two powers of $\rho$ in decay (resp. does not ameliorate the decay). The order of the symbol $W_{a b}^{\prime}$ is better when $a>0$ since, as soon as one takes at least one $\rho$-derivative of (3.1.1), the $W_{\infty}$ contribution disappears.

Proof of proposition 3.1.1: Let us check that the flow is global and that (3.1.7) holds. In particular, this will imply that, if $A$ is large enough, $\rho(t, s)$ stays larger than 1 , so that the decomposition (3.1.1) holds. Let $c>0, \delta_{0}>0$, be two small constants so that by (3.1.6)

$$
-\frac{\partial W_{\infty}}{\partial \theta}(\theta) \geq c \text { for any } \theta \text { with }|\theta| \leq \delta_{0}
$$

Denote by $T$ the supremum of those $t \in[0,+\infty$ [ such that the solution of (3.1.5) exists on $[0, t[$, and satisfies for any $t^{\prime} \in[0, t[$, any $s \in[1,2]$,

$$
\rho\left(t^{\prime}, s\right)^{2} \geq \frac{1}{2}(A s)^{2}+c t^{\prime} \text { and }\left|\theta\left(t^{\prime}, s\right)\right| \leq \delta_{0} .
$$


To check that $T=+\infty$ if $A \geq A_{0}$ for a large enough $A_{0}$, we just need to see that there is $C_{0}>0$, such that (3.1.15) implies

$$
C_{0}^{2}\left(A^{2}+t^{\prime}\right) \geq \rho\left(t^{\prime}, s\right)^{2} \geq \frac{3}{4}(A s)^{2}+2 c t^{\prime} \text { and }\left|\theta\left(t^{\prime}, s\right)\right| \leq \frac{\delta_{0}}{2},
$$

for any $t^{\prime} \in[0, t[$, any $s \in[1,2]$. Since, in the right hand side of the second equation (3.1.5),

$$
\rho\left(t^{\prime}, s\right)^{-1}\left|\frac{\partial \widetilde{W}}{\partial \rho}\left(\rho\left(t^{\prime}, s\right), \theta\left(t^{\prime}, s\right)\right)\right| \leq C\left(\frac{1}{2}(A s)^{2}+c t^{\prime}\right)^{-\frac{m}{2}-1}
$$

by (3.1.15), we get for $s \in[1,2], 0<t<T$,

$$
|\theta(t, s)| \leq \frac{s}{A}+\frac{C^{\prime}}{A^{m}} \leq \frac{\delta_{0}}{2}
$$

for large enough $A$ 's. It follows from (3.1.14), (3.1.1), (3.1.15) and the first equation in (3.1.5) that

$$
\frac{d}{d t} \rho(t, s)^{2} \geq 2 c-C\left(\frac{1}{2}(A s)^{2}+c t\right)^{-m / 2},
$$

for any $s \in[1,2], t \in[0, T[$. Since $m>2$, this implies

$$
\rho(t, s)^{2} \geq(A s)^{2}+2 c t-C^{\prime}(A s)^{-m+2},
$$

which gives the lower bound in the first estimate (3.1.16) for large enough $A$. The upper bound follows from (3.1.11). We have thus established global existence and (3.1.7).

To prove (3.1.8), we notice that (3.1.5) and (3.1.7) imply for $t \leq t^{\prime}$

$$
\left|\theta(t, s)-\theta\left(t^{\prime}, s\right)\right| \leq C\left(A^{2}+t\right)^{-\frac{m}{2}}
$$

Consequently, $\theta_{\infty}(s)=\lim _{t \rightarrow+\infty} \theta(t, s)$ exists and satisfies the last estimate in (3.1.8). We set

$$
\lambda(s)=-2 \frac{\partial W_{\infty}}{\partial \theta}\left(\theta_{\infty}(s)\right)
$$

Since, for $A$ large enough, $\left|\theta_{\infty}(s)\right| \leq \delta_{0}$ for any $s \in[1,2]$, we deduce from (3.1.14) the first formula in (3.1.8). Let us prove that there are numbers $B_{j}$ such that, for any $j \in \mathbb{N}$, any $t \geq 0$, any $s \in[1,2]$,

$$
\left|\frac{\partial^{j}}{\partial s^{j}}\left[\rho(t, s)^{2}\right]\right| \leq B_{j}\left(A^{2}+t\right),\left|\frac{\partial^{j}}{\partial s^{j}} \theta(t, s)\right| \leq \frac{B_{j}}{A} .
$$

Notice that if such estimates hold for derivatives of order up to $j-1,(3.1 .12),(3.1 .13)$ and (3.1.7) give for

$$
w_{1}(t, s)=\left|\partial_{s}^{j}\left[\rho(t, s)^{2}\right]\right|, w_{2}(t, s)=\left|\partial_{s}^{j} \theta(t, s)\right|,
$$

estimates

$$
w_{1}(t, s) \leq w_{1}(0, s)+C \int_{0}^{t}\left(A^{2}+t^{\prime}\right)^{-\frac{m}{2}-1} w_{1}\left(t^{\prime}, s\right) d t^{\prime}+C \int_{0}^{t} w_{2}\left(t^{\prime}, s\right) d t^{\prime}+\tilde{B}_{j-1}(t+1)
$$


and

$$
\begin{aligned}
w_{2}(t, s) \leq w_{2}(0, s) & +C \int_{0}^{t}\left(A^{2}+t^{\prime}\right)^{-\frac{m}{2}-2} w_{1}\left(t^{\prime}, s\right) d t^{\prime} \\
& +C \int_{0}^{t}\left(A^{2}+t^{\prime}\right)^{-\frac{m}{2}-1} w_{2}\left(t^{\prime}, s\right) d t^{\prime}+\tilde{B}_{j-1} \int_{0}^{t}\left(A^{2}+t^{\prime}\right)^{-\frac{m}{2}-1} d t^{\prime},
\end{aligned}
$$

where $\tilde{B}_{j-1}$ is a constant depending only on $B_{1}, \ldots, B_{j-1}$, and where we have used that in the last terms of (3.1.12), (3.1.13), the decay of $W_{a b}^{\prime}, W_{a b}^{\prime \prime}$ compensates the growth of $\partial_{s}^{\alpha} \rho^{2}$. If $B_{j}$ is large enough relatively to $\tilde{B}_{j-1}$, and if $A$ is large enough relatively to the constant $C$ in the right hand side of (3.1.20), (3.1.21), one deduces by bootstrap from these inequalities that (3.1.19) holds at rank $j$ (using that $m>2$ ).

It follows from (3.1.5), (3.1.7) and estimates (3.1.19) that, for any $s$, any $t \leq t^{\prime}$,

$$
\left|\partial_{s}^{j}\left[\theta(t, s)-\theta\left(t^{\prime}, s\right)\right]\right| \leq C_{j}\left(A^{2}+t\right)^{-\frac{m}{2}} .
$$

We conclude that $\theta_{\infty}$ is smooth and that $\partial_{s}^{j}\left[\theta_{\infty}(s)-\frac{s}{A}\right]=O\left(A^{-m}\right)$. Plugging this information in (3.1.18) and using the second condition in (3.1.6), we get the estimate of $\partial \lambda / \partial s$ in (3.1.8), taking $A$ large enough. Moreover, (3.1.22) shows that $\partial_{s}^{j}\left[\theta(t, s)-\theta_{\infty}(s)\right]=O\left(\left(A^{2}+t\right)^{-m / 2}\right)$. This, together with time derivative estimates for $\rho, \theta$ coming from (3.1.5), shows that the second formula in (3.1.10) holds. To get the first one, compute from (3.1.11), $\rho(t, s)^{2}=(A s)^{2}+\lambda(s) t+$ $\sigma(t, s)$ with

$$
\sigma(t, s)=-2 \int_{0}^{t}\left[\frac{\partial W_{\infty}}{\partial \theta}\left(\theta\left(t^{\prime}, s\right)\right)-\frac{\partial W_{\infty}}{\partial \theta}\left(\theta_{\infty}(s)\right)\right] d t^{\prime}-2 \int_{0}^{t} \frac{\partial \widetilde{W}}{\partial \theta}\left(\rho\left(t^{\prime}, s\right), \theta\left(t^{\prime}, s\right)\right) d t^{\prime} .
$$

Since $m>2$, it follows from the estimates of $\sigma_{2}$ in (3.1.9), (3.1.19) and (3.1.5) that

$$
\left|\partial_{t}^{\alpha} \partial_{s}^{\beta} \sigma(t, s)\right| \leq C_{\alpha, \beta}\left(A^{2}+t\right)^{-\alpha} .
$$

This implies the first formula in (3.1.9) and concludes the proof.

We denote by $\widetilde{\Lambda}_{0}^{A}$ the submanifold of the characteristic variety of $i \partial_{t}+\mathrm{Op}^{\mathrm{W}}(W)$ given by

$$
\widetilde{\Lambda}_{0}^{A}=\left\{(t, x ; \tau, \xi) ; t=0, \tau=W(x, \xi),(x, \xi) \in \Lambda_{0}^{A}\right\} \subset T^{*} \mathbb{R}^{2} .
$$

The flow-out of $\widetilde{\Lambda}_{0}^{A}$ by the Hamiltonian vector field of the symbol $-\tau+W(x, \xi)$ is given by

$$
\widetilde{\Lambda}^{A}=\left\{(t, x ; \tau, \xi) ; \tau=W(x, \xi),(x, \xi) \in \Lambda_{t}^{A}, t \geq 0\right\},
$$

where $\Lambda_{t}^{A} \subset T^{*} \mathbb{R}$ is the image of $\Lambda_{0}^{A}$ by the flow of (3.1.4) at time $t$. If we denote by (3.1.10) this flow expressed in polar coordinates, we get

$$
\Lambda_{t}^{A}=\{(\rho(t, s) \cos \theta(t, s), \rho(t, s) \sin \theta(t, s)) ; s \in[1,2]\} .
$$

Our next task will be to show that for large enough $A, t,(3.1 .25)$ may be written as the graph of a function. 
Define $Y(+\infty, s)=\lambda(s)^{1 / 2} \cos \theta_{\infty}(s)$. To fix ideas, we suppose from now on that $\partial \lambda / \partial s$, which has fixed sign on $[1,2]$ by $(3.1 .8)$, is positive (this corresponds, according to (3.1.18), to the case when $\frac{\partial^{2} W_{\infty}}{\partial \theta^{2}}(0)<0$ in $\left.(3.1 .6)\right)$. Consequently,

$$
C^{-1} A^{-1} \leq \frac{\partial \lambda}{\partial s}(s) \leq C A^{-1}, s \in[1,2] .
$$

By these inequalities and (3.1.19),

$$
\begin{aligned}
\frac{\partial}{\partial s} Y(+\infty, s) & =\frac{\lambda^{\prime}(s)}{2 \sqrt{\lambda(s)}} \cos \theta_{\infty}(s)-\sqrt{\lambda(s)} \theta_{\infty}^{\prime}(s) \sin \theta_{\infty}(s) \\
& \geq \frac{1}{2} C^{-3 / 2} A^{-1}+O\left(A^{-2}\right)
\end{aligned}
$$

uniformly for $s \in[1,2]$. We fix from now on $A$ larger than the constant $A_{0}$ of proposition 3.1.1, and large enough so that the above lower bound is strictly positive and so that $\frac{3}{A} \geq \theta_{\infty}(s) \geq \frac{1}{2 A}$ for any $s \in[1,2]$ (using (3.1.8)). Then $s \rightarrow Y(+\infty, s)$ is a strictly increasing diffeomorphism from $[1,2]$ onto $[Y(+\infty, 1), Y(+\infty, 2)]$. Fix a small positive number $\delta$ and define

$$
\begin{aligned}
\mu_{1}^{\prime}=Y(+\infty, 1+\delta) & <Y(+\infty, 1+2 \delta)<\mu_{1}=Y(+\infty, 1+3 \delta) \\
& <\mu_{2}=Y(+\infty, 2-3 \delta)<Y(+\infty, 2-2 \delta)<\mu_{2}^{\prime}=Y(+\infty, 2-\delta) .
\end{aligned}
$$

By (3.1.10), there are symbols $\hat{\sigma}_{1}, \hat{\sigma}_{2}$ of order -1 in $t$ such that the flow of (3.1.4) may be written

$$
\begin{aligned}
& X(t, s) \stackrel{\text { def }}{=} \rho(t, s) \cos \theta(t, s)=\sqrt{t} \lambda(s)^{1 / 2} \cos \theta_{\infty}(s)\left(1+\hat{\sigma}_{1}(t, s)\right) \\
& \Xi(t, s) \stackrel{\text { def }}{=} \rho(t, s) \sin \theta(t, s)=\sqrt{t} \lambda(s)^{1 / 2} \sin \theta_{\infty}(s)\left(1+\hat{\sigma}_{2}(t, s)\right) .
\end{aligned}
$$

If we set $Y(t, s)=\frac{1}{\sqrt{t}} X(t, s)=Y(+\infty, s)\left(1+\hat{\sigma}_{1}(t, s)\right)$, for $t \geq t_{0}$ with $t_{0}$ large enough, $s \rightarrow Y(t, s)$ is a diffeomorphism from $[1,2]$ onto its image. Set $\mu_{1}(t)=Y(t, 1+2 \delta), \mu_{2}(t)=Y(t, 2-2 \delta)$. Then $\mu_{1}(t)=Y(+\infty, 1+2 \delta)+O(1 / t), \mu_{2}(t)=Y(+\infty, 2-2 \delta)+O(1 / t)$, so that (3.1.27) implies that for $t \geq t_{0}$ large enough and some $\nu>0$,

$$
\left.\left[\mu_{1}, \mu_{2}\right] \subset\left[\mu_{1}(t), \mu_{2}(t)\right] \subset\left[\mu_{1}^{\prime}+\nu, \mu_{2}^{\prime}-\nu\right] \subset\left[\mu_{1}^{\prime}, \mu_{2}^{\prime}\right] \subset\right] Y(t, 1), Y(t, 2)[.
$$

Proposition 3.1.2 Assume that $A$ has been fixed as above. There is $t_{0} \geq 2$ and a smooth function $(t, y) \rightarrow Z(t, y)$, defined on $\left[t_{0},+\infty\left[\times\left[\mu_{1}^{\prime}, \mu_{2}^{\prime}\right]\right.\right.$, with values in a compact subset of $] 0,+\infty[$, such that $\partial_{t}^{\alpha} \partial_{y}^{\beta} Z(t, y)=O\left(t^{-\alpha}\right)$ and, for any $t \geq t_{0}$,

$$
\Lambda_{t}^{A} \cap \pi^{-1}\left(\left[\mu_{1}^{\prime} \sqrt{t}, \mu_{2}^{\prime} \sqrt{t}\right]\right)=\left\{\left(x, \sqrt{t} Z\left(t, \frac{x}{\sqrt{t}}\right)\right) ; \mu_{1}^{\prime} \sqrt{t} \leq x \leq \mu_{2}^{\prime} \sqrt{t}\right\},
$$

where $\pi:(x, \xi) \rightarrow x$.

Proof: By (3.1.29), for $\frac{x}{\sqrt{t}} \in\left[\mu_{1}^{\prime}, \mu_{2}^{\prime}\right]$, we may solve the equation $\frac{x}{\sqrt{t}}=Y(t, s)$ in $s$. The solution may be written $s=g(t, x / \sqrt{t})$ where $g:\left[t_{0},+\infty\left[\times\left[\mu_{1}^{\prime}, \mu_{2}^{\prime}\right] \rightarrow[1,2]\right.\right.$ is a smooth function with $\partial_{t}^{\alpha} \partial_{y}^{\beta} g(t, y)=O\left(t^{-\alpha}\right)$. Plugging this expression in the second formula (3.1.28), we get 
$\xi=\sqrt{t} Z(t, x / \sqrt{t})$ for a function $Z$ satisfying the conclusions of the statement. Notice that $Z(t, y)$ stays larger than a fixed positive constant, for $t \geq t_{0}$ large enough, $y \in\left[\mu_{1}^{\prime}, \mu_{2}^{\prime}\right]$, since in the second formula (3.1.28), $\theta_{\infty}(s) \sim A^{-1}$.

Proposition 3.1.2 shows that for $t \geq t_{0}, \Lambda_{t}^{A}$ is the graph of a function above $\left[\mu_{1}^{\prime} \sqrt{t}, \mu_{2}^{\prime} \sqrt{t}\right]$, so that

$$
\widetilde{\Lambda}^{A} \cap \pi^{-1}\left(\left\{(t, x) ; t \geq t_{0}, \mu_{1}^{\prime} \leq \frac{x}{\sqrt{t}} \leq \mu_{2}^{\prime}\right\}\right)
$$

is also the graph of a function of $(t, x)$. Since $\widetilde{\Lambda}^{A}$ is Lagrangian, there is a smooth function

$$
\psi:\left\{(t, x) ; t \geq t_{0}, \mu_{1}^{\prime} \sqrt{t} \leq x \leq \mu_{2}^{\prime} \sqrt{t}\right\} \rightarrow \mathbb{R}
$$

such that for any $(t, x)$ in the domain of definition

$$
\partial_{x} \psi(t, x)=\sqrt{t} Z\left(t, \frac{x}{\sqrt{t}}\right), \partial_{t} \psi(t, x)=W\left(x, \sqrt{t} Z\left(t, \frac{x}{\sqrt{t}}\right)\right) .
$$

\subsection{Construction of an approximate solution}

We assume from now on that the constants $A$ and $t_{0}$ have been fixed large enough, as in the statement of proposition 3.1.2. Our objective is to prove the following:

Proposition 3.2.1 Let $\mu_{1}(t), \mu_{2}(t)$ be defined as in (3.1.29) and set $K(t)=\left[\mu_{1}(t), \mu_{2}(t)\right], K=$ $(K(t))_{t \geq t_{0}}$. Let $N$ be a fixed integer. There is a sequence $b_{j}$ of elements of the class $\Sigma_{K}^{-j}$ of definition 1.2.2, $0 \leq j \leq N-1$, and a smooth function $(t, x) \rightarrow r_{N}(t, x)$ from $\left[t_{0},+\infty[\times \mathbb{R}\right.$ to $\mathbb{C}$, satisfying for any $\alpha, \beta$

$$
\left|\partial_{t}^{\alpha} \partial_{x}^{\beta} r_{N}(t, x)\right| \leq C_{\alpha \beta} t^{-\frac{1}{4}-N-\alpha-\frac{\beta}{2}-1}(\log t)^{\alpha+\beta+2 N}\left(1+\frac{|x|}{\sqrt{t}}\right)^{-N},
$$

such that if $b=\sum_{j=0}^{N-1} b_{j}$,

$$
\left(i \partial_{t}+\mathrm{Op}^{\mathrm{W}}(W)\right)\left[e^{i \psi(t, x)} b(t, x)\right]=e^{i \psi(t, x)} r_{N}(t, x)
$$

and $\left\|b_{0}(t, \cdot)\right\|_{L^{2}}$ is a positive constant independent of $t$.

We shall prove the proposition solving transport equations. Define for $x \in\left[\mu_{1}^{\prime} \sqrt{t}, \mu_{2}^{\prime} \sqrt{t}\right]$

$$
U(t, x)=-\left(\partial_{\xi} W\right)\left(x, \partial_{x} \psi(t, x)\right)
$$

and consider transport equations of the form

$$
\frac{\partial b}{\partial t}+U(t, x) \frac{\partial b}{\partial x}+\frac{1}{2}\left(\partial_{x} U(t, x)\right) b=\frac{1}{t^{2}} f
$$


where $f$ is a given source term and $t \geq t_{0}$. Since the Hamiltonian flow of $\tau-W(x, \xi)$ foliates $\widetilde{\Lambda}^{A}$, we may write for any $s \in[1,2]$ that the integral curves $t \rightarrow(X(t, s), \Xi(t, s))$, given by (3.1.28), satisfy for $t \geq t_{0}$,

$$
\begin{aligned}
\Xi(t, s) & =\partial_{x} \psi(t, X(t, s)) \\
\frac{d}{d t} X(t, s) & =U(t, X(t, s)) .
\end{aligned}
$$

We denote by $X^{-1}(t, x)$ the inverse of the diffeomorphism $X(t, \cdot)$. By definition of $\mu_{1}(t), \mu_{2}(t)$, $X^{-1}(t, \cdot)$ sends the interval $\left[\sqrt{t} \mu_{1}(t), \sqrt{t} \mu_{2}(t)\right]$ onto $[1+2 \delta, 2-2 \delta]$, and (3.1.29) shows that $X^{-1}(t, \cdot)$ is defined on $\left[\sqrt{t} \mu_{1}, \sqrt{t} \mu_{2}\right]$ for any $t \geq t_{0}$.

Lemma 3.2.2 (i) There is a constant $C>0$ such that for any $t \geq t^{\prime} \geq t_{0}$, any $s \in[1+2 \delta, 2-2 \delta]$,

$$
C^{-1}\left(\frac{t^{\prime}}{t}\right)^{1 / 2} \leq \exp \left[-\int_{t^{\prime}}^{t}\left(\partial_{x} U\right)(\tau, X(\tau, s)) d \tau\right] \leq C\left(\frac{t^{\prime}}{t}\right)^{1 / 2} .
$$

(ii) For any $(\alpha, \beta) \in \mathbb{N} \times \mathbb{N}$, there is $C_{\alpha \beta}>0$ such that, for any $t, \tau$ in $\left[t_{0},+\infty[\right.$, any $x \in$ $\left[\sqrt{t} \mu_{1}(t), \sqrt{t} \mu_{2}(t)\right]$,

$$
\begin{aligned}
\left|\partial_{t}^{\alpha} \partial_{x}^{\beta} U(t, x)\right| & \leq C_{\alpha \beta} t^{-\frac{1}{2}-\alpha-\frac{\beta}{2}} \\
\left|\partial_{t}^{\alpha} \partial_{x}^{\beta}\left[\left(\partial_{x} U\right)\left(\tau, X\left(\tau, X^{-1}(t, x)\right)\right)\right]\right| & \leq C_{\alpha \beta} \tau^{-1} t^{-\alpha-\frac{\beta}{2}} .
\end{aligned}
$$

Proof: (i) Let $\gamma_{0}$ be a smooth given function, compactly supported in $] \sqrt{t_{0}} \mu_{1}\left(t_{0}\right), \sqrt{t_{0}} \mu_{2}\left(t_{0}\right)[$ and consider the solution of

$$
\begin{aligned}
\frac{\partial \gamma}{\partial t}+U(t, x) \frac{\partial \gamma}{\partial x}+\frac{1}{2}\left(\partial_{x} U(t, x)\right) \gamma & =0 \\
\gamma\left(t_{0}, x\right) & =\gamma_{0}(x)
\end{aligned}
$$

Since

$$
\frac{d}{d t} \gamma(t, X(t, s))=-\frac{1}{2}\left(\partial_{x} U\right)(t, X(t, s)) \gamma(t, X(t, s))
$$

for any $t \geq t_{0}, x \rightarrow \gamma(t, x)$ is compactly supported in $] \sqrt{t} \mu_{1}(t), \sqrt{t} \mu_{2}(t)[$ and, since the equation is on divergence form,

$$
\int \gamma\left(t_{0}, x\right)^{2} d x=\int \gamma(t, x)^{2} d x
$$

for any $t \geq t_{0}$. By (3.2.9) we may write

$$
\gamma(t, X(t, s)) \exp \left[\frac{1}{2} \int_{t_{0}}^{t}\left(\partial_{x} U\right)(\tau, X(\tau, s)) d \tau\right]=\gamma\left(t_{0}, X\left(t_{0}, s\right)\right),
$$

so that

$$
\int \gamma(t, x)^{2} d x=\int \gamma\left(t_{0}, X\left(t_{0}, X^{-1}(t, x)\right)\right)^{2} \exp \left[-\int_{t_{0}}^{t}\left(\partial_{x} U\right)\left(\tau, X\left(\tau, X^{-1}(t, x)\right)\right) d \tau\right] d x .
$$


Using (3.2.10) to express the left hand side and changing variables in the right hand one, we get

$$
\int \gamma_{0}(x)^{2} d x=\int \gamma_{0}\left(X\left(t_{0}, s\right)\right)^{2}\left|\frac{\partial X}{\partial s}(t, s)\right| \exp \left[-\int_{t_{0}}^{t}\left(\partial_{x} U\right)(\tau, X(\tau, s)) d \tau\right] d s .
$$

Taking $\gamma_{0}(x)=\frac{1}{\sqrt{\epsilon}} \chi\left(\frac{x-X\left(t_{0}, a\right)}{\epsilon}\right)$, where $\left.\left.\int \chi(z)^{2} d z=1, a \in[1,2], \epsilon \in\right] 0,1\right]$, we obtain when $\epsilon \rightarrow 0$

$$
\Gamma(a)=\left|\frac{\partial X}{\partial s}(t, a)\right| \exp \left[-\int_{t_{0}}^{t}\left(\partial_{x} U\right)(\tau, X(\tau, a)) d \tau\right]
$$

where $\Gamma(a)$ is some positive continuous function of $a$. Since by (3.1.26), (3.1.28), $\left|\frac{\partial X}{\partial s}(t, a)\right| \sim \sqrt{t}$, we get (3.1.27) in the special case $t^{\prime}=t_{0}$. The general case follows by division.

(ii) The first inequality in (3.2.7) follows from (3.2.3), (3.1.32), the fact that $W$ is a symbol of order zero, and the bounds of $Z$ and its derivatives given in proposition 3.1.2.

The left hand side of the second inequality in (3.2.7) has modulus bounded in terms of expressions

$$
\left|\left(\partial_{x}^{p+1} U\right)\left(\tau, X\left(\tau, X^{-1}(t, x)\right)\right) \| \partial_{t}^{\alpha_{1}} \partial_{x}^{\beta_{1}} X\left(\tau, X^{-1}(t, x)\right)\right| \cdots\left|\partial_{t}^{\alpha_{p}} \partial_{x}^{\beta_{p}} X\left(\tau, X^{-1}(t, x)\right)\right|,
$$

where $\alpha_{1}+\cdots+\alpha_{p}=\alpha, \beta_{1}+\cdots+\beta_{p}=\beta, \alpha_{j}+\beta_{j} \geq 1$. By (3.2.3) and (3.1.32), for any $q \in \mathbb{N}$,

$$
\left|\partial_{x}^{q} U(\tau, x)\right|=\left|\frac{\partial^{q}}{\partial x^{q}}\left[\left(\partial_{\xi} W\right)\left(x, \sqrt{\tau} Z\left(\tau, \frac{x}{\sqrt{\tau}}\right)\right)\right]\right|=O\left(\tau^{-\frac{1}{2}-\frac{q}{2}}\right),
$$

when $x$ is replaced by $X(\tau, s), s \in[1+2 \delta, 2-2 \delta]$, since $Z$ stays between two positive constants as well as $\frac{1}{\sqrt{\tau}} X(\tau, s)$. Moreover, by (3.1.28),

$$
\left|\partial_{t}^{\alpha_{j}} \partial_{x}^{\beta_{j}} X\left(\tau, X^{-1}(t, x)\right)\right|=O\left(\sqrt{\tau} t^{-\alpha_{j}-\frac{\beta_{j}}{2}}\right)
$$

This gives the second upper bound in (3.2.7) and concludes the proof.

Lemma 3.2.3 (i) Let $c:] \sqrt{t_{0}} \mu_{1}\left(t_{0}\right), \sqrt{t_{0}} \mu_{2}\left(t_{0}\right)[\rightarrow \mathbb{C}$ be a smooth compactly supported function. The solution $b_{0}$, defined for $t \geq t_{0}$, of the equation

$$
\begin{aligned}
\frac{\partial b_{0}}{\partial t}(t, x)+U(t, x) \frac{\partial b_{0}}{\partial x}(t, x)+\frac{1}{2}\left(\partial_{x} U(t, x)\right) b_{0}(t, x) & =0 \\
b_{0}(t, x) & =c(x),
\end{aligned}
$$

is an element of $\Sigma_{K}^{0}$. Moreover, for any $t \geq t_{0},\left\|b_{0}(t, \cdot)\right\|_{L^{2}}=\|c\|_{L^{2}}$.

(ii) Let $j \in \mathbb{N}^{*}, c_{j-1}$ a given element of $\Sigma_{K}^{-(j-1)}$, defined for $t \geq t_{0}$. The equation

$$
\frac{\partial b_{j}}{\partial t}(t, x)+U(t, x) \frac{\partial b_{j}}{\partial x}(t, x)+\frac{1}{2}\left(\partial_{x} U(t, x)\right) b_{j}(t, x)=\frac{(\log t)^{2}}{t^{2}} c_{j-1}(t, x)
$$

has a solution $b_{j} \in \Sigma_{K}^{-j}$ defined for $t \geq t_{0}$. 
Proof: (i) Writing the equation under form (3.2.9), we get for $t \geq t_{0}$ and $\frac{x}{\sqrt{t}} \in\left[\mu_{1}(t), \mu_{2}(t)\right]$

$$
b_{0}(t, x)=c\left(X\left(t_{0}, X^{-1}(t, x)\right)\right) E\left(t_{0}, t, x\right)
$$

with

$$
E\left(t^{\prime}, t, x\right)=\exp \left[-\frac{1}{2} \int_{t^{\prime}}^{t}\left(\partial_{x} U\right)\left(\tau, X\left(\tau, X^{-1}(t, x)\right)\right) d \tau\right]
$$

It follows from the assumption on $c$ and from the definition of $\mu_{1}(t), \mu_{2}(t)$, that $b_{0}(t, x)$ is supported for $x / \sqrt{t}$ in $K(t)=\left[\mu_{1}(t), \mu_{2}(t)\right]$. By (3.2.6), we get that $\left|b_{0}(t, x)\right| \leq C t^{-1 / 4}$. We may write $\partial_{x}^{\beta} E\left(t^{\prime}, t, x\right)$ in terms of expressions

$$
\prod_{j=1}^{q}\left(-\frac{1}{2} \int_{t^{\prime}}^{t} \partial_{x}^{\beta_{j}}\left[\left(\partial_{x} U\right)\left(\tau, X\left(\tau, X^{-1}(t, x)\right)\right)\right] d \tau\right) E\left(t^{\prime}, t, x\right)
$$

with $\beta_{1}+\cdots+\beta_{q}=\beta, \beta_{j} \geq 1$. By (3.2.7) the absolute value of each integral is bounded from above by $C\left|\log \frac{t}{t^{\prime}}\right| t^{-\beta_{j} / 2}$ so that, taking (3.2.6) into account,

$$
\left|\partial_{x}^{\beta} E\left(t^{\prime}, t, x\right)\right| \leq C\left(\frac{t}{t^{\prime}}\right)^{-1 / 4} C\left\langle\log \frac{t}{t^{\prime}}\right\rangle^{\beta} t^{-\beta / 2} .
$$

We deduce from this and (3.2.11) that $b_{0}(t, x)$ satisfies estimates (1.2.9) with $j=\alpha=0$. Using (3.2.12) and (3.2.7) we obtain that estimates (1.2.9) with $\alpha>0$ are also valid, so that $b_{0}$ belongs to $\Sigma_{K}^{0}$. The fact that the $L^{2}$-norm of $b_{0}$ is conserved has been already checked in (3.2.10).

(ii) We write equation (3.2.13) under the form

$$
\frac{\partial}{\partial t} b_{j}(t, X(t, s))+\frac{1}{2}\left(\partial_{x} U\right)(t, X(t, s)) b_{j}(t, X(t, s))=\frac{(\log t)^{2}}{t^{2}} c_{j-1}(t, X(t, s)) .
$$

We solve this equation writing

$$
b_{j}(t, x)=\tilde{b}_{j}\left(t, X^{-1}(t, x)\right)
$$

with

$$
\tilde{b}_{j}(t, s)=-\int_{t}^{+\infty} \frac{(\log \tau)^{2}}{\tau^{2}} c_{j-1}(\tau, X(\tau, s)) \exp \left[\frac{1}{2} \int_{t}^{\tau}\left(\partial_{x} U\right)\left(\tau^{\prime}, X\left(\tau^{\prime}, s\right)\right) d \tau^{\prime}\right] d \tau .
$$

By (3.2.6), the exponential inside the integral is $O\left((t / \tau)^{-1 / 4}\right)$ and by $(1.2 .9), c_{j-1}(\tau, X(\tau, s))=$ $O\left(\tau^{-\frac{1}{4}-(j-1)}(\log \tau)^{2(j-1)}\right)$. This shows that the integral in (3.2.18) converges and that

$$
\left|\tilde{b}_{j}(t, s)\right| \leq C t^{-j-\frac{1}{4}}(\log t)^{2 j} .
$$

We get a solution vanishing when $t \rightarrow+\infty$, supported at time $t$ in the same interval as $c_{j-1}(t, \cdot)$. Let us write $\partial_{x}^{\beta} b_{j}(t, x)$ as a linear combination of expressions

$$
\int_{t}^{+\infty} \frac{(\log \tau)^{2}}{\tau^{2}} \partial_{x}^{\beta_{1}}\left[c_{j-1}\left(\tau, X\left(\tau, X^{-1}(t, x)\right)\right)\right] \partial_{x}^{\beta_{2}} E(\tau, t, x) d \tau
$$


with $\beta_{1}+\beta_{2}=\beta$. By (3.2.11) and (1.2.9)

$$
\left|\partial_{x}^{\beta_{1}}\left[c_{j-1}\left(\tau, X\left(\tau, X^{-1}(t, x)\right)\right)\right]\right| \leq C \tau^{-\frac{1}{4}-(j-1)} t^{-\beta_{1} / 2}(\log \tau)^{\beta_{1}+2(j-1)} .
$$

Using (3.2.16), we conclude that (3.2.19) is bounded from above by $C t^{-\frac{1}{4}-\frac{\beta}{2}-j}(\log t)^{\beta+2 j}$ for any $j \geq 1$. This gives (1.2.9) with $\alpha=0$. One recovers the estimates for $\alpha>0$ using equation (3.2.13) and (3.2.7). This concludes the proof.

Proof of proposition 3.2.1: If $b$ is an element of $\Sigma_{K}^{0}$ with $K(t)=\left[\mu_{1}(t), \mu_{2}(t)\right]$, it follows from proposition 1.2.3 applied with $m=0, j=0$ that, using notation (3.2.3),

$$
\begin{array}{r}
\left(i \partial_{t}+\mathrm{Op}^{\mathrm{W}}(W)\right)\left(e^{i \psi(t, x)} b(t, x)\right)=e^{i \psi(t, x)}\left[-\partial_{t} \psi(t, x)+W\left(x, \partial_{x} \psi(t, x)\right)\right] b(t, x) \\
+i e^{i \psi(t, x)}\left[\partial_{t} b(t, x)+U(t, x) \partial_{x} b(t, x)+\frac{1}{2} \partial_{x} U(t, x) b(t, x)-\frac{(\log t)^{2}}{t^{2}} L(b)(t, x)\right] \\
+e^{i \psi(t, x)} r(t, x),
\end{array}
$$

where $L$ is a linear operator acting from $\Sigma_{K}^{-j}$ to itself for any $j$, and $r$ satisfies (1.2.11). By (3.1.32) the first term in the right hand side vanishes. We look for $b$ as a sum $b=\sum_{j=0}^{N-1} b_{j}$ with $b_{j} \in \Sigma^{-j}$. We take for $b_{0}$ the solution of (3.2.12) with some non trivial initial data $c$ at time $t=t_{0}$, compactly supported inside $] \mu_{1}\left(t_{0}\right) \sqrt{t_{0}}, \mu_{2}\left(t_{0}\right) \sqrt{t_{0}}[$. We next solve (3.2.13) with $j=1$ and $c_{0}=L\left(b_{0}\right) \in \Sigma_{K}^{0}$. We obtain by lemma 3.2.3 an element $b_{1} \in \Sigma_{K}^{-1}$. Iterating the process, we construct recursively $b_{2}, \ldots, b_{N-1}$. The term $\frac{(\log t)^{2}}{t^{2}} L\left(b_{N-1}\right)$ satisfies estimates of type (3.2.1) as well as $r$, because of (1.2.11). This concludes the proof.

\subsection{Proof of the theorem}

This subsection will be devoted to the proof of theorem 1.1.3. Let $W \in \mathcal{S}^{0}(\mathbb{R} \times \mathbb{R})$ be a time independent symbol satisfying conditions (3.1.1), (3.1.2), (3.1.6). Let us construct a time dependent symbol $V$ in $\mathcal{S}^{0}\left(\mathbb{S}^{1} \times \mathbb{R} \times \mathbb{R}\right)$ satisfying equation (2.2.2) up to some remainder. The right hand side of that equation is

$$
\left.M_{0}(V)\right|_{t=0}+\left.\Omega(V)\right|_{t=0},
$$

where $M_{0}$ is given by (2.1.3) and, for a given $N$ and a family $\Omega_{j}, 1 \leq j \leq k_{N-1}$, of $j$-linear maps sending $\mathcal{S}^{m_{1}}\left(\mathbb{S}^{1} \times \mathbb{R} \times \mathbb{R}\right) \times \cdots \times \mathcal{S}^{m_{j}}\left(\mathbb{S}^{1} \times \mathbb{R} \times \mathbb{R}\right)$ to $\mathcal{S}^{m_{1}+\cdots+m_{j}-2}\left(\mathbb{S}^{1} \times \mathbb{R} \times \mathbb{R}\right)$,

$$
\Omega(V)-\sum_{j=1}^{k_{N-1}} \Omega_{j}(V, \ldots, V) \in \mathcal{S}^{-2 N}\left(\mathbb{S}^{1} \times \mathbb{R} \times \mathbb{R}\right) .
$$

Lemma 3.3.1 Let $N \in \mathbb{N}, W \in \mathcal{S}^{0}(\mathbb{R} \times \mathbb{R})$. One may find for $j=0, \ldots, N-1$ symbols $V_{j} \in \mathcal{S}^{-2 j}\left(\mathbb{S}^{1} \times \mathbb{R} \times \mathbb{R}\right)$ such that if $V=\sum_{j=0}^{N-1} V_{j}$

$$
W-\left.M_{0}(V)\right|_{t=0}-\left.\Omega(V)\right|_{t=0} \in \mathcal{S}^{-2 N}(\mathbb{R} \times \mathbb{R}) .
$$


Proof: Define for $U \in \mathcal{S}^{m}(\mathbb{R} \times \mathbb{R})$ an element $E U \in \mathcal{S}^{m}\left(\mathbb{S}^{1} \times \mathbb{R} \times \mathbb{R}\right)$ by $(E U)(t, x, \xi)=$ $U(\rho(-t) \cdot(x, \xi))$. Then $\left.\left(M_{0} E U\right)\right|_{t=0}=U$. Using (3.3.2), we decompose

$$
\Omega(V)=\sum_{j=1}^{N-1} \widetilde{\Omega}_{j}\left(\left(V_{k}\right)_{0 \leq k \leq j-1}\right)+R\left(V_{0}, \ldots, V_{N-1}\right)
$$

where $\widetilde{\Omega}_{j}$ belongs to $\mathcal{S}^{-2 j}\left(\mathbb{S}^{1} \times \mathbb{R} \times \mathbb{R}\right)$ and depends only on $V_{k}$ for $k<j$, and $R$ is in $\mathcal{S}^{-2 N}\left(\mathbb{S}^{1} \times\right.$ $\mathbb{R} \times \mathbb{R})$. We define recursively

$$
V_{0}=E W, V_{j}=-E\left[\left.\widetilde{\Omega}_{j}\left(V_{0}, \ldots, V_{j-1}\right)\right|_{t=0}\right], 1 \leq j \leq N-1 .
$$

We obtain equation (3.3.3).

Let us apply proposition 2.2.2 with the symbol $V$ associated to $W$ by lemma 3.3.1. We obtain a symbol $m \in \mathcal{S}^{0}\left(\mathbb{S}^{1} \times \mathbb{R} \times \mathbb{R}\right)$ such that

$$
\begin{aligned}
e^{-i t P_{0}} \mathrm{Op}^{\mathrm{W}}(m)^{*} & {\left[i \partial_{t}+P_{0}+\mathrm{Op}^{\mathrm{W}}(V)\right] \mathrm{Op}^{\mathrm{W}}(m) e^{i t P_{0}} } \\
= & i \partial_{t}+\mathrm{Op}^{\mathrm{W}}(W)+\mathrm{Op}^{\mathrm{W}}\left(U_{2}\right)+e^{-i t P_{0}} \mathrm{Op}^{\mathrm{W}}\left(U_{1}\right) e^{i t P_{0}} \\
& +e^{-i t P_{0}} \mathrm{Op}^{\mathrm{W}}(S) e^{i t P_{0}} \circ\left(i \partial_{t}\right)+\left(i \partial_{t}\right) \circ e^{-i t P_{0}} \mathrm{Op}^{\mathrm{W}}(S) e^{i t P_{0}},
\end{aligned}
$$

where $U_{1}$ and $U_{2}$ are in $\mathcal{S}^{-2 N}\left(\mathbb{S}^{1} \times \mathbb{R} \times \mathbb{R}\right)$ and $S$ belongs to $\mathcal{S}^{-2 N-2}\left(\mathbb{S}^{1} \times \mathbb{R} \times \mathbb{R}\right)$.

The main remaining step in the proof of theorem 1.1.3 is given by the following:

Proposition 3.3.2 Let $s \in \mathbb{R}_{+}, N \in \mathbb{N}$ with $N>s+2$. There is a smooth function $(t, x) \rightarrow$ $w_{a}(t, x)$ defined for $t \geq t_{0}, x \in \mathbb{R}$, compactly supported in $x$ at fixed $t$, and a constant $C>0$ such that, for $t \geq t_{0}$,

$$
\begin{aligned}
\left\|w_{a}(t, \cdot)\right\|_{\mathcal{H}^{s}} & \geq C^{-1} t^{s / 2}, \\
\left\|\left(i \partial_{t}+P_{0}+\mathrm{Op}^{\mathrm{W}}(V)\right) w_{a}(t, \cdot)\right\|_{\mathcal{H}^{s}} & \leq C t^{\frac{s}{2}-N+1} .
\end{aligned}
$$

Proof: Let $b$ be the function given by proposition 3.2 .1 and set

$$
v_{a}(t, x)=e^{i \psi(t, x)} b(t, x) .
$$

By $(3.2 .2)$

$$
\left(i \partial_{t}+\mathrm{Op}^{\mathrm{W}}(W)\right) v_{a}=e^{i \psi(t, x)} r_{N}(t, x)
$$

with $r_{N}$ satisfying estimate (3.2.1). We set

$$
w_{a}(t, x)=\mathrm{Op}^{\mathrm{W}}(m(t, \cdot)) e^{i t P_{0}} v_{a}(t, x) .
$$

We apply the operator (3.3.4) to $v_{a}$. We get

$$
\begin{aligned}
\mathrm{Op}^{\mathrm{W}}(m)^{*}\left[i \partial_{t}+P_{0}+\mathrm{Op}^{\mathrm{W}}(V)\right] w_{a}= & e^{i t P_{0}}\left[e^{i \psi(t, x)} r_{N}\right]+e^{i t P_{0}} \mathrm{Op}^{\mathrm{W}}\left(U_{2}\right) v_{a} \\
& +\mathrm{Op}^{\mathrm{W}}\left(U_{1}\right) e^{i t P_{0}} v_{a}+\mathrm{Op}^{\mathrm{W}}(S) e^{i t P_{0}}\left(i \partial_{t} v_{a}\right) \\
& +e^{i t P_{0}} i \partial_{t}\left[e^{-i t P_{0}} \mathrm{Op}^{\mathrm{W}}(S) e^{i t P_{0}} v_{a}\right] .
\end{aligned}
$$


Proposition 1.2.3 applied to a symbol $a$ of order $s-2 N$ shows that $\mathrm{Op}^{\mathrm{W}}(a) v_{a}=e^{i \psi(t, x)}(c(t, x)+$ $r(t, x))$, where $c$ is supported for $x / \sqrt{t}$ staying in a fixed compact set and satisfies $|c(t, x)| \leq$ $C t^{-\frac{1}{4}+\frac{s}{2}-N}$, and where $r$ satisfies (1.2.11) with an arbitrary large $N$. We conclude that

$$
\left\|v_{a}(t, \cdot)\right\|_{\mathcal{H}^{s-2 N}} \leq C t^{\frac{s}{2}-N},\left\|\partial_{t} v_{a}(t, \cdot)\right\|_{\mathcal{H}^{s-2 N-2}} \leq C t^{\frac{s}{2}-N}
$$

Using these inequalities, together with the fact that $e^{i t P_{0}}$ is isometric, and that $U_{1}, U_{2}$ are of order $-2 N$, and $S$ of order $-2 N-2$, we rewrite (3.3.8) as

$$
\mathrm{Op}^{\mathrm{W}}(m)^{*}\left[i \partial_{t}+P_{0}+\mathrm{Op}^{\mathrm{W}}(V)\right] w_{a}=g
$$

with $\|g(t, \cdot)\|_{\mathcal{H}^{s}} \leq C t^{\frac{s}{2}-N}(\log t)^{2 N}$. Using (2.2.4), we may construct a symbol $q$ of order zero such that $\mathrm{Op}^{\mathrm{W}}(q) \mathrm{Op}^{\mathrm{W}}(m)^{*}=I d-\mathrm{Op}^{\mathrm{W}}(r)$, where $r$ is of order $-2 N-2$. We get

$$
\left(i \partial_{t}+P_{0}+\mathrm{Op}^{\mathrm{W}}(V)\right) w_{a}=\tilde{g}
$$

with

$$
\tilde{g}=\mathrm{Op}^{\mathrm{W}}(q) g(t, \cdot)+\mathrm{Op}^{\mathrm{W}}(r)\left(i \partial_{t}+P_{0}+\mathrm{Op}^{\mathrm{W}}(V)\right) w_{a} .
$$

Since by (3.3.7)

$$
\left\|\partial_{t} w_{a}\right\|_{\mathcal{H}^{s-2 N-2}} \leq C\left[\left\|\partial_{t} v_{a}\right\|_{\mathcal{H}^{s-2 N-2}}+\left\|v_{a}\right\|_{\mathcal{H}^{s-2 N}}\right]
$$

we get $\|\tilde{g}(t, \cdot)\|_{\mathcal{H}^{s}} \leq C t^{\frac{s}{2}-N}(\log t)^{2 N}$. This implies the second estimate in (3.3.5).

We are left with showing that if $b$ is conveniently chosen in (3.3.6), the lower bound in (3.3.5) holds. Denoting now by $q$ a symbol of order 0 such that $\mathrm{Op}^{\mathrm{W}}(q) \mathrm{Op}^{\mathrm{W}}(m)=I d+\mathrm{Op}^{\mathrm{W}}(r)$ with $r$ of order $-2 N$, we may write

$$
\begin{aligned}
\left\|v_{a}(t, \cdot)\right\|_{\mathcal{H}^{s}} & =\left\|e^{i t P_{0}} v_{a}(t, \cdot)\right\|_{\mathcal{H}^{s}} \\
& \leq\left\|\mathrm{Op}^{\mathrm{W}}(q) \mathrm{Op}^{\mathrm{W}}(m) e^{i t P_{0}} v_{a}(t, \cdot)\right\|_{\mathcal{H}^{s}}+\left\|\mathrm{Op}^{\mathrm{W}}(r) e^{i t P_{0}} v_{a}(t, \cdot)\right\|_{\mathcal{H}^{s}} \\
& \leq C\left\|w_{a}(t, \cdot)\right\|_{\mathcal{H}^{s}}+C\left\|v_{a}(t, \cdot)\right\|_{\mathcal{H}^{s-2 N}} .
\end{aligned}
$$

Because of (3.3.9), we see that we just need to get a lower bound for

$$
\left\|v_{a}(t, \cdot)\right\|_{\mathcal{H}^{s}} \sim\left\|\mathrm{Op}^{\mathrm{W}}\left(\left(x^{2}+\xi^{2}+1\right)^{s / 2}\right) v_{a}(t, \cdot)\right\|_{L^{2}} .
$$

By (3.3.6) and proposition 1.2.3,

$$
\mathrm{Op}^{\mathrm{W}}\left(\left(x^{2}+\xi^{2}+1\right)^{s / 2}\right) v_{a}(t, x)=e^{i \psi(t, x)}[\tilde{b}(t, x)+\tilde{c}(t, x)]
$$

where

$$
\begin{aligned}
\tilde{b}(t, x) & =\left(x^{2}+\left(\partial_{x} \psi(t, x)\right)^{2}+1\right)^{s / 2} b(t, x) \\
|c(t, x)| & \leq C t^{-\frac{1}{4}+\frac{s}{2}} \frac{(\log t)}{t}\left\langle\frac{x}{\sqrt{t}}\right\rangle^{-M},
\end{aligned}
$$

where $M>1$ and where we have used that on the support of $b(t, \cdot),|x| \sim \sqrt{t}$ and $\left|\partial_{x} \psi(t, x)\right| \sim \sqrt{t}$. These properties show that

$$
\left\|v_{a}(t, \cdot)\right\|_{\mathcal{H}^{s}} \geq c t^{s / 2}\|b(t, \cdot)\|_{L^{2}}+o\left(t^{s / 2}\right), t \rightarrow+\infty .
$$


We just need to check that $\|b(t, \cdot)\|_{L^{2}} \geq c>0$ for large enough $t$. This follows from proposition 3.2.1, as $b=\sum_{j=0}^{N-1} b_{j}$ with $\left\|b_{0}(t, \cdot)\right\|_{L^{2}}$ equal to a positive constant and $\left\|b_{j}(t, \cdot)\right\|_{L^{2}}=$ $O\left(t^{-j}(\log t)^{2 j}\right), t \rightarrow+\infty$ when $j \geq 1$. This concludes the proof of the proposition.

Proof of theorem 1.1.3: Let $w_{a}$ be the function of proposition 3.3.2, that we extend smoothly for all $t \geq 0$. We look for an exact solution $u$ of (1.1.5) as $u=w_{a}+\tilde{u}$, where $\tilde{u}$ satisfies

$$
\left(i \partial_{t}+P_{0}+\mathrm{Op}^{\mathrm{W}}(V)\right) \tilde{u}=-\tilde{g}
$$

with $\tilde{g}$ given by (3.3.10). By (3.3.5), $\|\tilde{g}\|_{\mathcal{H}^{s}} \leq C t^{\frac{s}{2}-N+1}$. Using the notation introduced in the statement of lemma 1.2.1 we may take

$$
\tilde{u}(t, \cdot)=\int_{t}^{+\infty} S\left(t, t^{\prime}\right) \tilde{g}\left(t^{\prime}, \cdot\right) d t^{\prime}
$$

and by $(1.2 .8)$

$$
\|\tilde{u}(t, \cdot)\|_{\mathcal{H}^{s}} \leq C \int_{t}^{+\infty}\left\langle t-t^{\prime}\right\rangle^{s / 2}\left\langle t^{\prime}\right\rangle^{-N+\frac{s}{2}+1} d t^{\prime} \leq C\langle t\rangle^{-N+s+2}
$$

if $N$ has been taken strictly larger than $s+2$. Consequently

$$
\|u(t, \cdot)\|_{\mathcal{H}^{s}} \geq\left\|w_{a}(t, \cdot)\right\|_{\mathcal{H}^{s}}-\|\tilde{u}(t, \cdot)\|_{\mathcal{H}^{s}} \geq \frac{1}{2} C^{-1} t^{s / 2}
$$

when $t \rightarrow+\infty$, using (3.3.5). This concludes the proof.

\section{References}

[1] J.-M. Barbaroux and A. Joye: Expectation values of observables in time dependent quantum mechanics, J. Statist. Phys. 90 (1998), no. 5-6, 1225-1249.

[2] J. Bourgain: Growth of Sobolev norms in linear Schrödinger equations with quasi-periodic potential, Comm. Math. Phys. 204 (1999), no. 1, 207-247.

[3] J. Bourgain: On growth of Sobolev norms in linear Schrödinger equations with smooth time dependent potential, J. Anal. Math. 77 (1999), 315-348.

[4] J.-M. Delort: Growth of Sobolev norms of solutions of linear Schrödinger equations on some compact manifolds, Int. Math. Res. Notices (2010), no. 12, 2305-2328.

[5] P. Duclos, O. Lev and P. Štovíček: On the energy growth of some periodically driven quantum systems with shrinking gaps in the spectrum, J. Stat. Phys. 130 (2008), no. 1, 169-193.

[6] H. Eliasson and S. Kuksin: On reducibility of Schrödinger equations with quasiperiodic potentials, Comm. Math. Phys. 286 (2009), no. 1, 125-135.

[7] D. Fang and Q. Zhang: On growth of Sobolev norms in linear Schrödinger equations with time dependent Gevrey potential, preprint, (2010), 34 pp. 
[8] B. Grébert and L. Thomann: KAM for the quantum harmonic oscillator, preprint, (2010), $52 \mathrm{pp}$.

[9] J. S. Howland: Floquet operators with singular spectrum III, Ann. Inst. H. Poincaré Phys. Théor. 69 (1998), no. 2, 265-273.

[10] G. Nenciu: Adiabatic theory: Stability of systems with increasing gaps, Ann. Inst. H. Poincaré Phys. Théor. 67 (1997), no. 4, 411-424.

[11] M. A. Shubin: Pseudodifferential operators and spectral theory, Second edition. SpringerVerlag, Berlin, 2001. xii+288 pp.

[12] W.-M. Wang: Logarithmic bounds on Sobolev norms for time dependent linear Schrödinger equations, Comm. Partial Differential Equations 33 (2008), no. 10-12, 2164-2179.

[13] W.-M. Wang: Pure point spectrum of the Floquet Hamiltonian for the quantum harmonic oscillator under time quasi-periodic perturbations, Comm. Math. Phys. 277 (2008), no. 2, 459-496.

[14] W.-M. Wang: Bounded Sobolev norms for linear Schrödinger equations under resonant perturbations, J. Funct. Anal. 254 (2008), no. 11, 2926-2946. 